\title{
Time-Varying Systemic Risk: Evidence from a Dynamic Copula Model of CDS Spreads
}

\author{
Dong Hwan Oh
}

Duke University
Andrew J. Patton

Duke University

May 23, 2013

ERID Working Paper Number 167

This paper can be downloaded without charge from the Social Science Research Network Electronic Paper Collection:

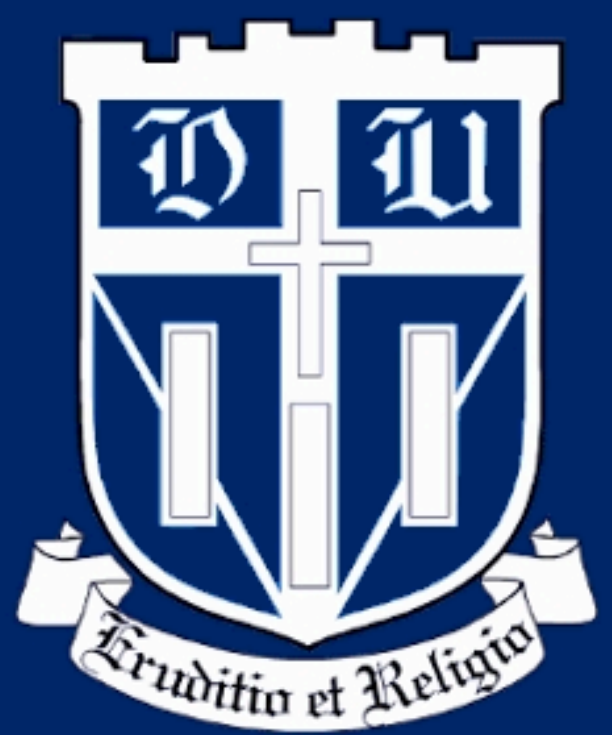




\title{
Time-Varying Systemic Risk: Evidence from a Dynamic Copula Model of CDS Spreads*
}

\author{
Dong Hwan Oh and Andrew J. Patton \\ Duke University
}

23 May 2013

\begin{abstract}
This paper proposes a new class of copula-based dynamic models for high dimension conditional distributions, facilitating the estimation of a wide variety of measures of systemic risk. Our proposed models draw on successful ideas from the literature on modeling high dimension covariance matrices and on recent work on models for general time-varying distributions. Our use of copula-based models enable the estimation of the joint model in stages, greatly reducing the computational burden. We use the proposed new models to study a collection of daily credit default swap (CDS) spreads on 100 U.S. firms over the period 2006 to 2012. We find that while the probability of distress for individual firms has greatly reduced since the financial crisis of 2008-09, the joint probability of distress (a measure of systemic risk) is substantially higher now than in the pre-crisis period.
\end{abstract}

Keywords: correlation, tail risk, financial crises, DCC.

J.E.L. codes: C32, C58, G01.

\footnotetext{
${ }^{*}$ We thank Drew Creal and seminar participants at Chicago, Duke, Monash, Montréal, NYU, UNC-Chapel Hill,
} UNSW and UTS for helpful comments. An appendix containing additional results for this paper is available at http://www.econ.duke.edu/ ap172/research.html. Contact address: Andrew Patton, Department of Economics, Duke University, 213 Social Sciences Building, Box 90097, Durham NC 27708-0097. Email: andrew.patton@duke.edu. 


\section{Introduction}

Systemic risk can be broadly defined as the risk of distress in a large number of firms or institutions. It represents an extreme event in two directions: a large loss (e.g., corresponding to a large left-tail realization for stock returns), across a large proportion of the firms. There are a variety of methods for studying risk and dependence for small collections of assets, see Patton (2012) for a review of copula-based approaches, but a relative paucity of methods for studying dependence between a large collection of assets, which is required for a general analysis of systemic risk.

Some existing methods for estimating systemic risk simplify the task by reducing the dimension of the problem to two: an individual firm and a market index. The "CoVaR" measure of Adrian and Brunnermeier (2009), for example, uses quantile regression to estimate a lower tail quantile (e.g., 0.05) of market returns conditional on a given firm having a returns equal to its lower tail quantile. The "marginal expected shortfall" proposed by Brownlees and Engle (2011) estimates the expected return on a firm conditional on the market return being below some low threshold. These methods have the clear benefit of being parsimonious, but by aggregating the "non firm $i$ " universe to a single market index, useful information about systemic risk may be missed. The objective of this paper is to provide models that can be used to handle large collections of variables, which enables the estimation of a wider variety of systemic risk measures.

We use Sklar's theorem (see Nelsen, 2006), with an extension to conditional distributions from Patton (2006), to decompose the conditional joint distribution of a collection of $N$ variables into their marginal distributions and a conditional copula:

$$
\mathbf{Y}_{t} \mid \mathcal{F}_{t-1} \sim \mathbf{F}_{t}=\mathbf{C}_{t}\left(F_{1 t}, \ldots, F_{N t}\right)
$$

We propose new models for the time-varying conditional copula, $\mathbf{C}_{t}$, that can be used to link models of the conditional marginal distributions (e.g., ARMA-GARCH models) to form a dynamic conditional joint distribution. Of central relevance to this paper are cases where $N$ is relatively large, around 50 to 250 . In such cases, models that have been developed for low dimension problems (say, $N<5$ ) are often not applicable, either because no generalization beyond the bivariate model exists, or because such generalizations are too restrictive (e.g., Archimedean copulas have just one 
or two free parameters regardless of $N$, which is clearly very restrictive in high dimensions), or because the obvious generalization of the bivariate case leads to a proliferation of parameters and unmanageable computational complexity. In high dimension applications, the challenge is to find a balance of flexibility and parsimony.

This paper makes two contributions. First, we propose a flexible and feasible model for capturing time-varying dependence in high dimensions. Our approach draws on successful ideas from the literature on dynamic modeling of high dimension covariance matrices and on recent work on models for general time-varying distributions. In particular, we combine the "GAS" model of Creal, et al. (2011, 2013), parameter restrictions and "variance targeting" ideas from Engle (2002) and Engle and Kelly (2012), and the factor copula model of Oh and Patton (2012) to obtain a flexible yet parsimonious dynamic model for high dimension conditional distributions. A realistic simulation study confirms that our proposed models and estimation methods have satisfactory properties for relevant sample sizes.

Our second contribution is a detailed study of a collection of 100 daily credit default swap (CDS) spreads on U.S. firms. The CDS market has expanded enormously over the last decade, growing 40-fold from $\$ 0.6$ trillion of gross notional principal in 2001 to $\$ 25.9$ trillion at the end of 2011 according to the International Swaps and Derivatives Association (ISDA), yet it has received relatively little attention in the econometrics literature. (Interest is growing, however, see Conrad, et al. (2011), Lucas, et al. (2011), Creal, et al. (2012) and Christoffersen, et al. (2013) for recent work on CDS data.) We use our model of CDS spreads to provide insights into systemic risk, as CDS spreads are tightly linked to the health of the underlying firm. We find that systemic risk rose during the financial crisis, unsurprisingly. More interestingly, we also find that systemic risk remains high relative to the pre-crisis period, even though idiosyncratic risk has fallen.

The remainder of the paper is structured as follows. Section 2 presents a dynamic copula model for high dimension applications, and Section 3 presents a simulation study for the proposed model. In Section 4 we present estimation results for various models of CDS spreads. Section 5 presents estimates of time-varying systemic risk, and Section 6 concludes. Technical details are presented in the appendix, and an internet appendix contains some additional results. 


\section{A dynamic copula model for high dimensions}

In this section we describe our approach for capturing dynamics in the dependence between a relatively large number of variables. A review of alternative methods from the (small) extant literature is presented in Section 2.3. We consider a class of data generating processes (DGPs) that allow for time-varying conditional marginal distributions, e.g., dynamic conditional means and variances, and also possibly time-varying higher-order moments:

$$
\begin{aligned}
\mathbf{Y}_{t} & \equiv\left[Y_{1 t}, \ldots, Y_{N t}\right]^{\prime} \\
\text { where } Y_{i t} & =\mu_{i t}\left(\phi_{i, 0}\right)+\sigma_{i t}\left(\phi_{i, 0}\right) \eta_{i t}, \quad i=1,2, \ldots, N \\
\eta_{i t} \mid \mathcal{F}_{t-1} & \sim F_{i t}\left(\phi_{i, 0}\right)
\end{aligned}
$$

where $\mu_{i t}$ is the conditional mean of $Y_{i t}, \sigma_{i t}$ is the conditional standard deviation, and $F_{i t}\left(\phi_{i, 0}\right)$ is a parametric distribution with zero mean and unit variance. We will denote the parameters of the marginal distributions as $\phi \equiv\left[\phi_{1}^{\prime}, \ldots, \phi_{N}^{\prime}\right]^{\prime}$, the parameters of the copula as $\gamma$, and the vector of all parameters as $\boldsymbol{\theta} \equiv\left[\boldsymbol{\phi}^{\prime}, \gamma^{\prime}\right]^{\prime}$. We assume that $F_{i t}$ is continuous and strictly increasing, which fits our empirical application, though this assumption can be relaxed. The information set is taken to be $\mathcal{F}_{t}=\sigma\left(\mathbf{Y}_{t}, \mathbf{Y}_{t-1}, \ldots\right)$. Define the conditional probability integral transforms of the data as:

$$
U_{i t} \equiv F_{i t}\left(\frac{Y_{i t}-\mu_{i t}\left(\phi_{i, 0}\right)}{\sigma_{i t}\left(\phi_{i, 0}\right)} ; \phi_{i, 0}\right), \quad i=1,2, \ldots, N
$$

Then the conditional copula of $\mathbf{Y}_{t} \mid \mathcal{F}_{t-1}$ is equal to the conditional distribution of $\mathbf{U}_{t} \mid \mathcal{F}_{t-1}$ :

$$
\mathbf{U}_{t} \mid \mathcal{F}_{t-1} \sim \mathbf{C}_{t}\left(\gamma_{0}\right)
$$

By allowing for a time-varying conditional copula, the class of DGPs characterized by equations (2) to (4) is a generalization of those considered by Chen and Fan (2006), for example, however the cost of this flexibility is the need to specify parametric marginal distributions. In contrast, Chen and Fan (2006), Rémillard (2010) and Oh and Patton (2013) allow for nonparametric estimation of the marginal distributions. The parametric margin requirement arises as the asymptotic distribution theory for a model with nonparametric margins and a time-varying copula is not yet available in the literature. We attempt to mitigate this requirement in our empirical work by using flexible models 
for the marginal distributions, and conducting goodness-of-fit tests to verify that they provide a satisfactory fit to the data.

\subsection{Factor copulas}

In high dimension applications a critical aspect of any model is imposing some form of dimension

reduction. A widely-used method to achieve this in economics and finance is to use some form of factor structure. Oh and Patton (2012) propose using a factor model with flexible distributions to obtain a flexible class of "factor copulas." A one-factor version of their model is the copula for the (latent) vector random variable $\mathbf{X}_{t} \equiv\left[X_{1 t}, \ldots, X_{N t}\right]^{\prime}$ implied by the following structure:

$$
\begin{aligned}
X_{i t} & =\lambda_{i t}\left(\gamma_{\lambda}\right) Z_{t}+\varepsilon_{i t}, \quad i=1,2, \ldots, N \\
\text { where } Z_{t} & \sim F_{z t}\left(\gamma_{z}\right), \quad \varepsilon_{i t} \sim i i d F_{\varepsilon t}\left(\gamma_{\varepsilon}\right), Z \Perp \varepsilon_{i} \forall i
\end{aligned}
$$

where $F_{z t}\left(\gamma_{z}\right)$ and $F_{\varepsilon t}\left(\gamma_{\varepsilon}\right)$ are flexible parametric univariate distributions for the common factor and the idiosyncratic variables respectively, and $\lambda_{i t}\left(\gamma_{\lambda}\right)$ is a potentially time-varying weight on the common factor. The conditional joint distribution for $\mathbf{X}_{t}$ can be decomposed into its conditional marginal distributions and its conditional copula via Sklar's theorem (see Nelsen (2006)) for conditional distributions, see Patton (2006):

$$
\mathbf{X}_{t} \sim \mathbf{F}_{x t}=\mathbf{C}_{t}\left(G_{1 t}(\gamma), \ldots, G_{N t}(\gamma) ; \gamma\right)
$$

where $\gamma \equiv\left[\gamma_{z}^{\prime}, \gamma_{\varepsilon}^{\prime}, \gamma_{\lambda}^{\prime}\right]^{\prime}$. Note that the marginal distributions of $\mathbf{X}_{t}$ need not be the same as the marginal distributions of the observed data. Only the copula of these variables, denoted $\mathbf{C}_{t}(\gamma)$, is used as a model for the copula of the observable data $\mathbf{Y}_{t}$. If we impose that the marginal distributions of the observable data are also driven by the factor structure in equation (5), then this becomes a standard factor model for a vector of variables. However, Oh and Patton (2012) suggest imposing the factor structure only on the component of the multivariate model where dimension reduction is critical, namely the copula, and allow the marginal distributions to be modeled using a potentially different approach. In this case, the factor structure in equation (5) is used only for the copula that it implies, and this becomes a "factor copula" model. 
The copula implied by equation (5) is known in closed form for only a few particular combinations of choices of $F_{z}$ and $F_{\varepsilon}$ (the most obvious example being where both of these distributions are Gaussian, in which case the implied copula is also Gaussian). For general choices of $F_{z}$ and $F_{\varepsilon}$ the copula of $\mathbf{X}$ will not be known in closed form, and thus the copula likelihood is not known in closed form. Numerical methods can be used to overcome this problem. Oh and Patton (2013) propose simulated method of moments-type estimation of the unknown parameters, however their approach is only applicable when the conditional copula is constant. A key objective of this paper is to allow the conditional copula to vary through time and so an alternate estimation approach is required. We use a simple numerical integration method, described in Appendix A, to overcome the lack of closed-form likelihood. This numerical integration exploits the fact that although the copula is $N$-dimensional, we need only integrate out the common factor, which is one-dimensional in the structure above.

Dynamics in the factor copula model in equation (5) arise by allowing the loadings on the common factor, $\lambda_{i t}$, to vary through time, and/or by allowing the distributions of the common factor and the idiosyncratic variables to change through time. For example, holding $F_{z t}$ and $F_{\varepsilon t}$ fixed, an increase in the factor loadings corresponds to an increase in the level of overall dependence (e.g., rank correlation) between the variables. Holding the factor loadings fixed, an increase in the thickness of the tails of the distribution of the common factor increases the degree of tail dependence. In the next section we describe how we model these dynamics.

\section{2 "GAS" dynamics}

An important feature of any dynamic model is the specification for how the parameters evolve through time. Some specifications, such as stochastic volatility models (see Shephard (2005) for example) and related stochastic copula models (see Hafner and Manner (2011) and Manner and Segers (2011)) allow the varying parameters to evolve as a latent time series process. Others, such as ARCH-type models for volatility (see Engle, 1982) and related models for copulas (see Patton (2006), Jondeau and Rockinger (2006), and Creal, et al. (2013) for example) model the varying parameters as some function of lagged observables. An advantage of the latter approach over the 
former, in particular for high dimension applications, is that it avoids the need to "integrate out" the innovation terms driving the latent time series processes.

Within the class of ARCH-type models ("observation driven", in the terminology of Creal, et al. (2013)), the question of which function of lagged observables to use as a forcing variable in the evolution equation for the varying parameter arises. For models of the conditional variance, an immediate choice is the lagged squared residual, as in the ARCH model, but for models with parameters that lack an obvious interpretation the choice is less clear. We adopt the generalized autoregressive score (GAS) model of Creal, et al. (2013) to overcome this problem. (Harvey (2013) and Harvey and Sucarrat (2012) propose a similar method for modeling time-varying parameters, which they call a "dynamic conditional score," or "DCS," model.) These authors propose using the lagged score of the density model (copula model, in our application) as the forcing variable. Specifically, for a copula with time-varying parameter $\boldsymbol{\delta}_{t}$, governed by fixed parameter $\boldsymbol{\gamma}$, we have:

$$
\text { Let } \begin{aligned}
\mathbf{U}_{t} \mid \mathcal{F}_{t-1} & \sim \mathbf{C}\left(\boldsymbol{\delta}_{t}(\boldsymbol{\gamma})\right) \\
\text { then } \boldsymbol{\delta}_{t} & =\boldsymbol{\omega}+B \boldsymbol{\delta}_{t-1}+A \mathbf{s}_{t-1} \\
\text { where } \mathbf{s}_{t-1} & =S_{t-1} \boldsymbol{\nabla}_{t-1} \\
\boldsymbol{\nabla}_{t-1} & =\frac{\partial \log \mathbf{c}\left(\mathbf{u}_{t-1} ; \boldsymbol{\delta}_{t-1}\right)}{\partial \boldsymbol{\delta}_{t-1}}
\end{aligned}
$$

and $S_{t}$ is a scaling matrix (e.g., the inverse Hessian or its square root). While this specification for the evolution of a time-varying parameter is somewhat arbitrary, Creal, et al. (2013) provide two motivations for it. Firstly, this model nests a variety of popular and successful existing models: GARCH (Bollerslev (1986)) for conditional variance; ACD (Engle and Russell (1998)) for models of trade durations (the time between consecutive high frequency observations); Davis, et al.'s (2003) model for Poisson counts. Secondly, the recursion above can be interpreted as the steepest ascent direction for improving the model's fit, in terms of the likelihood, given the current value of the model parameter $\boldsymbol{\delta}_{t}$, similar to numerical optimization algorithms such as the Gauss-Newton algorithm. Harvey (2013) further motivates this specification as an approximation to a filter for a model driven by a stochastic latent parameter, or an "unobserved components" model. 


\subsubsection{GAS dynamics for high dimension factor copulas}

We employ the GAS model to allow for time variation in the factor loadings in the factor copula implied by equation (5), but to keep the model parsimonious we impose that the parameters

governing the "shape" of the common and idiosyncratic variables $\left(\gamma_{z}\right.$ and $\left.\gamma_{\varepsilon}\right)$ are constant. We use the skewed $t$ distribution of Hansen (1994) as the model for $F_{z}$, and the (symmetric) standardized $t$ distribution as the model for $F_{\varepsilon}$. The skewed $t$ distribution has two shape parameters, a degrees of freedom parameter $\left(\nu_{z} \in(2, \infty]\right)$ and an asymmetry parameter $\left(\psi_{z} \in(-1,1)\right)$. This distribution simplifies to the standardized $t$ distribution when $\psi=0$. We impose symmetry on the distribution of the idiosyncratic variables for simplicity.

In the general GAS framework in equation (7), the $N$ time-varying factor loadings would have $N+2 N^{2}$ parameters governing their evolution, which represents an infeasibly large number for even moderate values of $N$. To keep the model parsimonious, we impose that the coefficient matrices ( $B$ and $A$ ) are diagonal with a common parameter on the diagonal, as in the DCC model of Engle (2002). To avoid the estimation of $N \times N$ scaling matrix we set $S_{t}=I$. This simplifies our model to be $($ in $\log s)$ :

$$
\log \lambda_{i t}=\omega_{i}+\beta \log \lambda_{i, t-1}+\alpha s_{i, t-1}, \quad i=1,2, \ldots, N
$$

where $s_{i t} \equiv \partial \log \mathbf{c}\left(\mathbf{u}_{t} ; \boldsymbol{\lambda}_{t}, \nu_{z}, \psi_{z}, \nu_{\varepsilon}\right) / \partial \lambda_{i t}$ and $\boldsymbol{\lambda}_{t} \equiv\left[\lambda_{1 t}, \ldots, \lambda_{N t}\right]^{\prime}$. The dynamic copula model implied by equations (5) and (8) thus contains $N+2$ parameters for the GAS dynamics, 3 parameters for the shape of the common and idiosyncratic variables, for a total of $N+5$ parameters.

\subsubsection{Equidependence vs. heterogeneous dependence}

To investigate whether we can further reduce the number of free parameters in this model we consider two restrictions of the model in equation (8), motivated by the "dynamic equicorrelation" model of Engle and Kelly (2012). If we impose that $\omega_{i}=\omega \forall i$, then the pair-wise dependence between each of the variables will be identical, leading to a "dynamic equidependence" model. (The copula implied by this specification is "exchangeable" in the terminology of the copula literature.) In this case we have only 6 parameters to estimate independent of the number of variables $N$, vastly 
reducing the estimation burden, but imposing a lot of homogeneity on the model.

An intermediate step between the fully flexible model in equation (8) and the equidependence model is to group the assets using some ex ante information (e.g., by industry for stock returns or CDS spreads) and impose homogeneity only within groups. This leads to a "block equidependence" model, with

$$
\begin{aligned}
X_{i t} & =\lambda_{g(i), t} Z_{t}+\varepsilon_{i t}, \quad i=1,2, \ldots, N \\
\log \lambda_{g, t} & =\omega_{g}+\beta \log \lambda_{g, t-1}+\alpha s_{g, t-1}, \quad g=1,2, \ldots, G
\end{aligned}
$$

where $g(i)$ is the group to which variable $i$ belongs, and $G$ is the number of groups. In this case the number of parameters to estimate in the copula model is $G+2+3$. In our empirical application we have $N=100$ and we consider grouping variables into $G=5$ industries, meaning this model has 10 parameters to estimate rather than 105. In our empirical analysis below, we compare these two restricted models $(G=1$ and $G=5)$ with the "heterogeneous dependence" model which allows a different factor for each variable $(G=N)$.

\subsubsection{A "variance targeting" method}

Estimating the fully flexible model above involves numerically searching over $N+5$ parameters, and for $N=100$ this represents quite a computational challenge. We propose a method to overcome this challenge by adapting an idea from the DCC model of Engle (2002). Specifically, we use a "variance targeting" (Engle and Mezrich (1996)) method to replace the constant $\omega_{i}$ in the GAS equation with a transformation of a sample dependence measure. The nature of our GAS specification means that the variance targeting approach needs to be modified for use here.

The evolution equation for $\lambda_{i t}$ in equation (8) can be re-written as

$$
\log \lambda_{i t}=E\left[\log \lambda_{i t}\right](1-\beta)+\beta \log \lambda_{i, t-1}+\alpha s_{i, t-1}
$$

using the result from Creal, et al. (2013) that $E_{t-1}\left[s_{i t}\right]=0$, and so $E\left[\log \lambda_{i t}\right]=\omega_{i} /(1-\beta)$. The proposition below provides a method for using sample rank correlations to obtain an estimate of $E\left[\log \lambda_{i t}\right]$, thus removing the need to numerically optimize over the intercept parameters, $\omega_{i}$. The proposition is based on the following assumption. 
Assumption 1 (a) The conditional copula of $\mathbf{Y}_{t} \mid \mathcal{F}_{t-1}$ is the time-varying factor copula given in equations (5) and (8).

(b) The process $\left\{\boldsymbol{\lambda}_{t}\right\}$ generated by equation (8) is strictly stationary.

(c) Let $\boldsymbol{\rho}_{t, X} \equiv \operatorname{vech}\left(\operatorname{RankCorr}_{t-1}\left[\mathbf{X}_{t}\right]\right)$. Then $\log \boldsymbol{\lambda}_{t}$ is a linear function of $\boldsymbol{\rho}_{t, X}$.

(d) Let $\rho_{i j, X} \equiv \operatorname{RankCorr}\left[X_{i}, X_{j}\right]$ and $\rho_{i j, X}^{L} \equiv \operatorname{Corr}\left[X_{i}, X_{j}\right]$. Then, for fixed values of $\left(\gamma_{z}, \gamma_{\varepsilon}\right)$, the mapping $\rho_{i j}=\varphi\left(\rho_{i j}^{L}\right)$ is strictly increasing.

Part (a) of this assumption makes explicit that the copula of the data is the GAS-factor copula model, and so the conditional copula of $\mathbf{Y}_{t} \mid \mathcal{F}_{t-1}$ is the same as that of $\mathbf{X}_{t} \mid \mathcal{F}_{t-1}$. Blasques, et al. (2012) which provide conditions under which univariate GAS models satisfy stationarity conditions; corresponding theoretical results for the multivariate case are not yet available in the literature, and thus in part (b) we simply assume that stationarity holds. Part (c) formalizes the applicability of a Taylor series expansion of the function mapping $\boldsymbol{\rho}_{t}$ to $\boldsymbol{\lambda}_{t}$. In practice this assumption will hold only approximately, and its applicability needs to be verified via simulation, which we discuss further in Section 3. Part (d) enables us to map rank correlations to linear correlations. Note that we can take $\left(\gamma_{z}, \gamma_{\varepsilon}\right)$ as fixed, as we call this mapping for each evaluation of the log-likelihood, which provides us with a value for $\left(\gamma_{z}, \gamma_{\varepsilon}\right)$. Importantly, this mapping can be computed prior to estimation, and then just called during estimation, rather than re-computed each time the likelihood function is evaluated.

Proposition 1 Let Assumption 1 hold, and denote the vech of the rank correlation matrix of the standardized residuals as $\overline{\boldsymbol{\rho}}_{\eta}^{S}$ and its sample analog as $\hat{\boldsymbol{\rho}}_{\eta}^{S}$. Then:

(i) $E\left[\log \boldsymbol{\lambda}_{t}\right]=H\left(\overline{\boldsymbol{\rho}}_{\eta}^{S}\right)$, where $H$ is defined in equation (27).

(ii) $\widehat{\log \boldsymbol{\lambda}}=H\left(\hat{\boldsymbol{\rho}}_{\eta}^{S}\right)$ is a GMM estimator of $E\left[\log \boldsymbol{\lambda}_{t}\right]$.

Part (i) of the above proposition provides the mapping from the population rank correlation of the standardized residuals to the mean of the (log) factor loadings, which is the basis for considering a variance-targeting type estimator. Part (ii) shows that the sample analog of this mapping can be interpreted as a standard GMM estimator. This is useful as it enables us to treat the estimation of the entire conditional joint distribution model as multi-stage GMM, and draw on results for such 
estimators to conduct inference, see White (1994), Engle and Sheppard (2001) and Gonçalves et al. (2013). The latter paper provides conditions under which a block bootstrap may be used to obtain valid standard errors on parameters estimated via multi-stage GMM. The resulting standard errors are not higher-order efficient, like some bootstrap inference methods, but they do enable us to avoid having to handle Hessian matrices of size on the order of $2 N \times 2 N$. Note that sample rank correlations cannot in general be considered as moment-based estimators, as they depend on the sample ranks of the observed data, and studying their estimation properties requires alternative techniques. However, we exploit the fact that the marginal distributions of the data are known up to an unknown parameter vector, and thus rank correlation can be computed as a sample moment of a nonlinear function of the data.

\subsection{Other models for dynamic, high dimension copulas}

As noted above, the literature contains relatively few models for dynamic, high dimension copulas. Exceptions to this are discussed here. Lucas, et al. (2011) combine GAS dynamics with a skewed $t$ copula to model ten sovereign CDS spreads. A similar model, though with an alternative skew $t$ specification and with Engle's (2002) DCC dynamics, is used by Christoffersen, et al. (2012, 2013). The former of these two papers analyzes equity returns on up to 33 national stock indices, while the latter studies weekly equity returns and CDS spreads on 233 North American firms (and is the largest time-varying copula model in the extant literature). Almeida et al. (2012) use "vine" copulas to model the dependence between 30 German stock return series, with dynamics captured through a stochastic volatility-type equation for the parameters of the copula. Stöber and Czado (2012) also use vine copulas, combined with a regime-switching model for dynamics, to model dependence between ten German stock returns.

\section{Simulation study}

This section presents an analysis of the finite sample properties of maximum likelihood estimation for factor copulas with GAS dynamics. Factor copulas do not have a closed-form likelihood, and we 
approximate the likelihood using some standard numerical integration methods, details of which can be found in Appendix A. Oh and Patton (2013) propose SMM-type estimation for factor copulas to overcome the lack of a closed-form likelihood, but a likelihood approach allows us to exploit the GAS model of Creal, et al. (2013) and so we pursue that here.

We consider three different copula models described for the Monte Carlo simulation: a dynamic equidependence model $(G=1)$, a dynamic block equidependence model $(G=10)$, and a dynamic heterogeneous dependence model $(G=N)$, all of them governed by:

$$
\begin{aligned}
X_{i t} & =\lambda_{g(i), t} Z_{t}+\varepsilon_{i t}, \quad i=1,2, \ldots, N \\
\log \lambda_{g, t} & =\omega_{g}+\beta \log \lambda_{g, t-1}+\alpha s_{g, t-1}, \quad g=1,2, \ldots, G \\
Z & \sim \operatorname{Skew} t\left(\nu_{z}, \psi_{z}\right) \\
\varepsilon_{i} & \sim \operatorname{iidt}\left(\nu_{\varepsilon}\right), \text { and } \varepsilon_{i} \Perp Z \forall i
\end{aligned}
$$

We set $N=100$ to match the number of series in our empirical application below. For simplicity, we impose that $\nu_{z}=\nu_{\varepsilon}$, and we estimate $\nu^{-1}$ rather than $\nu$, so that Normality is nested at $\nu^{-1}=0$ rather than $\nu \rightarrow \infty$. Broadly matching the parameter estimates we obtain in our empirical application, we set $\omega=0, \beta=0.98, \alpha=0.05, \nu=5$, and $\psi_{z}=0.1$ for the equidependence model. The block equidependence model uses the same parameters but sets $\omega_{1}=-0.03$ and $\omega_{10}=0.03$, and with $\omega_{2}$ to $\omega_{9}$ evenly spaced between these two bounds, and the heterogeneous dependence model similarly uses $\omega_{1}=-0.03$ and $\omega_{100}=0.03$, with $\omega_{2}$ to $\omega_{99}$ evenly spaced between these two bounds. Rank correlations implied by these values range from 0.1 to 0.7 . With these choices of parameter values and dependence designs, various dynamic dependence structures are covered, and asymmetric tail dependence, which is a common feature of financial data, is also allowed. We use a sample size of $T=500$ and we repeat each simulation 100 times.

The results for the equidependence model presented in Panel A of Table 1 reveal that the average estimated bias for all parameters is small, and the estimates are centered on true values. The results for the block equidependence model, presented in Panel B, are also satisfactory, and, as expected, the estimation error in the parameters is generally slightly higher for this more complicated model.

The heterogeneous dependence model is estimated using the variance targeting-type approach 
for the intercepts, $\omega_{i}$, described in Section 2.2.3, combined with numerical optimization for the remaining parameters. The internet appendix contains simulations that verify the applicability of Assumption 1 for this model, and the results presented in Panel C confirm that the approach leads to estimators with satisfactory finite-sample properties. (Panel C reports only every fifth intercept parameter, in the interests of space. The complete set of results is available in the internet appendix.) The standard errors on the estimated intercept parameters are approximately twice as large, on average, as in the block equidependence case, however this model has seven times as many parameters as the block equidependence (104 vs. 14) and so some loss in accuracy is inevitable. Importantly, all estimated parameters are approximately centered on their true values, confirming that the assumptions underlying Proposition 1 are applicable for this model.

\section{[INSERT TABLE 1 ABOUT HERE]}

\section{Data description and estimation results}

\subsection{CDS spreads}

We apply the dynamic copula model described in the previous section to daily credit default swap (CDS) spreads, obtained from Markit. In brief, a CDS is a contract in which the seller provides insurance to the buyer against any losses resulting from a default by the "reference entity" within some horizon. We focus on North American corporate CDS contracts, and the reference entities are thus North American firms. The CDS spread, usually measured in basis points and payable quarterly by the buyer to the seller, is the cost of this insurance. See Duffie and Singleton (2003) and Hull (2012) for more detailed discussions of CDS contracts, and see Barclays "CDS Handbook" (2010) for institutional details.

A key reason for interest in CDS contracts is sensitivity of CDS spreads to changes in market perceptions of the probability of default, see Conrad, et al. (2011), Creal, et al. (2012) and Christoffersen, et al. (2013) for recent empirical studies of implied default probabilities. Under some simplifying assumptions (such as a constant risk free rate and default hazard rate) see Carr 
and $\mathrm{Wu}$ (2011) for example, it can be shown that the CDS spread in basis points is:

$$
S_{i t}=100^{2} P_{i t}^{\mathbb{Q}} L_{i t}
$$

where $L_{i t}$ is the loss given default (sometimes shortened to "LGD," and often assumed to equal

0.6 for U.S. firms) and $P_{i t}^{\mathbb{Q}}$ is the implied probability of default. The same formula can also be obtained as a first-order approximation at $P_{i t}^{\mathbb{Q}} \approx 0$ for other more complicated pricing equations. This expression can be written in terms of the objective probability of default, $P_{i t}^{\mathbb{P}}$ :

$$
S_{i t}=100^{2} P_{i t}^{\mathbb{P}} \mathcal{M}_{i t} L_{i t}
$$

where $\mathcal{M}_{i t}$ is the market price of risk (stochastic discount factor). An increase in a CDS spread can be driven by an increase in the LGD, an increase in the market price of default risk for this firm, or an increase in the objective probability of default. Any one of these three effects is indicative of a worsening of the health of the underlying firm.

In the analysis below we work with the log-difference of CDS spreads, to mitigate their autoregressive persistence, and under this transformation we obtain:

$$
Y_{i t} \equiv \Delta \log S_{i t}=\Delta \log P_{i t}^{\mathbb{P}}+\Delta \log \mathcal{M}_{i t}+\Delta \log L_{i t}
$$

If the loss given default is constant then the third term above vanishes, and if we assume that the market price of risk is constant (as in traditional asset pricing models) or evolves slowly (for example, with a business cycle-type frequency) then daily changes in CDS spreads can be attributed primarily to changes in the objective probability of default. We will use this to guide our interpretation of the empirical results below, but we emphasize here that an increase in any of these three terms represents "bad news" for firm $i$, and so the isolation of the objective probability of default is not required for our interpretations to follow.

\subsection{Summary statistics}

Our sample period spans January 2006 to April 2012, a total of 1644 days. We study the 5-year CDS contract, which is the most liquid horizon (see Barclays (2010)), and we use "XR" ("no 
restructuring") CDS contracts, which became the convention for North America following the CDS market standardization in 2009 (the so-called "Big Bang"). To obtain a set of active, economically interesting, CDS data, we took all 125 individual firms in the CDS index covering our sample period (CDX Series 17). Of these, 90 firms had data that covered our entire sample period, and ten firms had no more than three missing observations. We use these 100 firms for our analysis. (Of the remaining 25 firms, six are not U.S.-based firms and one firm stopped trading because of a firm split. None of the firms defaulted over this sample period.) A plot of these CDS spreads is presented in Figure 1, which reveals that the average CDS spread was around 100 basis points (bps), and it varied from a low (averaged across firms) of 24 bps on February 22, 2007, to a high of 304 bps on March 9, 2009.

\section{[INSERT FIGURE 1 ABOUT HERE ]}

The levels of our CDS spread data are suggestive of a large autoregressive root, with the median first-order autocorrelation across all 100 series being 0.996 (the minimum is 0.990). Further, augmented Dickey-Fuller tests reject the null hypothesis of a unit root at the 0.05 level for only 12 series. Like interest rate time series, these series are unlikely to literally obey a random walk, as they are bounded below, however we model all series in log differences to avoid the need to consider these series as near unit root processes.

Table 2 presents summary statistics on our data. Of particular note is the positive skewness of the log-differences in CDS spreads (average skewness is 1.087, and skewness is positive for 89 out of 100 series) and the excess kurtosis (25.531 on average, and greater than 3 for all 100 firms). LjungBox tests for autocorrelation at up to the tenth lag find significant (at the 0.05 level) autocorrelation in 98 out of 100 of the log-differenced CDS spreads, and for 89 series significant autocorrelation is found in the squared log-differences. This motivates specifying models for the conditional mean and variance to capture this predictability.

\section{[ INSERT TABLE 2 ABOUT HERE ]}




\subsection{Conditional mean and variance models}

Daily log-differences of CDS spreads have more autocorrelation than is commonly found for daily stock returns (e.g., the average first-order autocorrelation is 0.161 ) and so the model for the conditional mean of our data needs more structure than the commonly-used constant model for daily stock returns. We use an $\mathrm{AR}(5)$ augmented with one lag of the market variable (an equal-weighted average of all 100 series), and we show below that this model passes standard specification tests:

$$
Y_{i t}=\phi_{0 i}+\sum_{j=1}^{5} \phi_{j i} Y_{i, t-j}+\phi_{m i} Y_{m, t-1}+e_{i t}
$$

For the market return we use the same model (omitting, of course, a repeat of the first lag of the market return). We need a model for the market return as we use the residuals from the market return model in our conditional variance specification.

Our model for the conditional variance is the asymmetric volatility model of Glosten, et al. (1993), the "GJR-GARCH" model. The motivation for the asymmetry in this model is that "bad news" about a firm increases its future volatility more than good news. For stock returns, bad news comes in the form of a negative residual. For CDS spreads, on the other hand, bad news is a positive residual, and so we reverse the direction of the indicator variable in the GJR-GARCH model to reflect this. In addition to the standard GJR-GARCH terms, we also include terms relating to the lagged market residual:

$$
\begin{aligned}
V_{t-1}\left[e_{i t}\right] \equiv & \sigma_{i t}^{2}=\omega_{i}+\beta_{i} \sigma_{i, t-1}^{2}+\alpha_{i} e_{i, t-1}^{2}+\delta_{i} e_{i, t-1}^{2} \mathbf{1}\left\{e_{i, t-1}>0\right\} \\
& +\alpha_{i m} e_{m, t-1}^{2}+\delta_{i m} e_{m, t-1}^{2} \mathbf{1}\left\{e_{m, t-1}>0\right\}
\end{aligned}
$$

Finally, we specify a model for the marginal distribution of the standardized residuals, $\eta_{i t}$. We use the skewed $t$ distribution of Hansen (1994), which allows for non-zero skewness and excess kurtosis:

$$
\eta_{i t} \equiv \frac{e_{i t}}{\sigma_{i t}} \sim \text { iid Skew } t\left(\nu_{i}, \psi_{i}\right)
$$

Table 3 summarizes the results of estimating the above models on the 100 time series. For the conditional mean model, we find strong significance of the first three AR lags, as well as the lagged 
market return. The conditional variance models reveal only mild statistical evidence of asymmetry in volatility, however the point estimates suggest that "bad news" (a positive residual) increases future volatility about $50 \%$ more than good news. The average estimated degrees of freedom parameter is 3.620, suggestive of fat tails, and the estimated skewness parameter is positive for 94 firms, and significantly different from zero for 41 of these, indicating positive skewness.

We now discuss goodness-of-fit tests for the marginal distribution specifications. We firstly use the Ljung-Box test to check the adequacy of these models for the conditional mean and variance, and we are able to reject the null of zero autocorrelation up to the tenth lag for only nine of the residual series, and only two of the squared standardized residual series. We conclude that these models provide a satisfactory fit to the conditional means and variances of these series. Next, we use the Kolmogorov-Smirnov test to investigate the fit of the skewed $t$ distribution for the standardized residuals, using 100 simulations to obtain critical values that capture the parameter estimation error, and we reject the null of correct specification for just eleven of the 100 firms. This is slightly higher than the level of the test (0.05), but we do not pursue the use of a more complicated marginal distribution model for those eleven firms in the interests of parsimony and comparability.

\section{[ INSERT TABLE 3 ABOUT HERE ]}

\subsection{The CDS "Big Bang"}

On April 8, 2009, the CDS market underwent changes driven by a move towards more standardized CDS contracts. Details of these changes are described in Markit (2009). It is plausible that the changes to the CDS market around the Big Bang changed the dynamics and distributional features of CDS time series, and we test for that possibility here. We do so by allowing the parameters of the mean, variance, and marginal distribution models to change on the date of the Big Bang, and we test the significance of these changes. We have 591 pre-break observations and 1053 post-break observations.

We find that the conditional mean parameters changed significantly (at the 0.05 level) for 39 
firms, and the conditional variance and marginal density shape parameters changed significantly for 66 firms. In what follows, the results we report are based on models that allow for a structural break in the mean, variance and distribution parameters. Given the prevalence of these changes, all of the copula models we consider allow for a break at the date of the Big Bang.

\subsection{Comparing models for the conditional copula}

The class of high dimension dynamic copula models described in Section 2 includes a variety of possible specifications: static vs. GAS dynamics; normal vs. skew $t$ - $t$ factor copulas; equidependence vs. block equidependence vs. heterogeneous dependence.

Table 4 presents results for six different dynamic models (a corresponding table for the six static copula models is available in the internet appendix). Bootstrap standard errors are presented in parentheses below the estimated parameters. (We use the stationary block bootstrap of Politis and Romano (1994) with an average block length of 120 days, applied to the log-difference of the CDS spreads, and we use 100 bootstrap replications.) Similar to other applications of GAS models (see, Creal et al. (2011, 2013)) we find strong persistence, with the $\beta$ parameter ranging from 0.85 to 0.99 . (Note that the $\beta$ parameter in GAS models plays the same role as $\alpha+\beta$ in a GARCH(1,1) model, see Example 1 in Creal, et al. (2013)). We also find that the inverse degrees of freedom parameters are greater than zero (i.e., the factor copula is not Normal), which we test formally below. We further find that the asymmetry parameter for the common factor is positive,

indicating greater dependence for joint upward moves in CDS spreads. This is consistent with financial variables being more correlated during bad times: for stock returns bad times correspond to joint downward moves, which have been shown in past work to be more correlated than joint upward moves, while for CDS spreads bad times correspond to joint upward moves.

\section{[ INSERT TABLE 4 ABOUT HERE ]}

Table 4 shows that the estimated degrees of freedom parameter for the common factor is larger than that for the idiosyncratic term. Oh and Patton (2012) show that when these two parameters differ the tail dependence implied by this factor copula is on the boundary: either zero $\left(\nu_{z}>\nu_{\varepsilon}\right)$ 
or one $\left(\nu_{z}<\nu_{\varepsilon}\right)$; only when these parameters are equal can tail dependence lie inside $(0,1)$. We test the significance of the difference between these two parameters by estimating a model with them imposed to be equal and then conducting a likelihood ratio test, the log-likelihoods from these two models are reported in Table 5 . The results strongly suggest that $\nu_{z}>\nu_{\varepsilon}$, and thus that extreme movements in CDS spreads are uncorrelated. The average gain in the log likelihood from estimating just this one extra parameter is around 200 points. This does not mean, of course, that "near extreme" movements must be uncorrelated, only that they are uncorrelated in the limit.

Table 5 also shows a comparison of the Skew $t$ - $t$ factor copula with the Normal copula, which is obtained by using a Normal distribution for both the common factor and the idiosyncratic factor. We see very clearly that the Normal copula performs worse than the Skew $t$ - $t$ factor copula, with the average gain in the log likelihood of the more flexible model being over 2000 points. This represents yet more evidence against the Normal copula model for financial time series; the Normal copula is simply too restrictive.

\section{[ INSERT TABLE 5 ABOUT HERE ]}

Finally, Table 5 compares the results from models with three different degrees of heterogeneity: equidependence vs. block equidependence vs. heterogeneous dependence. We see that the data support the more flexible models, with the block equidependence model improving on the equidependence model by around 200 points, and the heterogeneous model improving on the block equidependence model by around 800 points. It should be noted that our use of industry membership to form the "blocks" is just one method, and alternative grouping schemes may lead to better results. We do not pursue this possibility here.

Given the results in Table 5, our preferred model for the dependence structure of these 100 CDS spread series is a skew $t$ - $t$ factor copula, with separate degrees of freedom for the common and idiosyncratic variables, allowing for a separate loading on the common factor for each series (the "heterogeneous dependence" model) and allowing for dynamics using the GAS structure described in the previous section. Figure 2 presents the time-varying factor loadings implied by this model, and Figure 3 presents time-varying rank correlations. To summarize these results, Figure 
2 averages the loadings across all firms in the same industry, and Figures 3 averages all pair-wise correlations between firms in the same pairs of industries. (Thus the plotted factor loadings and rank correlations are smoother than any individual rank correlation plot.) Also presented in Figure 3 are 60-day rolling window rank correlations, again averaged across pairs of the firms in the same pair of industries. This figure reveals a secular increase in the correlation between CDS spreads, rising from around 0.1 in 2006 to around 0.5 in 2013. Interestingly, rank correlations do not appear to spike during the financial crisis, unlike individual volatilities and probabilities of default; rather they continue a mostly steady rise through the sample period.

\section{[ INSERT FIGURES 2 AND 3 ABOUT HERE ]}

\section{Time-varying systemic risk}

In this section we use the dynamic multivariate model presented above to obtain estimates of measures of systemic risk. A variety of measures of systemic risk have been proposed in the literature to date. One influential measure is "CoVaR," proposed by Adrian and Brunnermeier (2009), which uses quantile regression to estimate the lower tail (e.g., 0.05) quantile of market returns conditional on a given firm having a return equal to its lower tail quantile. This measure provides an estimate of how firm-level stress spills over to the market index. An alternative measure is "marginal expected shortfall" (MES) proposed by Brownlees and Engle (2011), which estimates the expected return on a firm conditional on the market return being below some low threshold. Segoviano and Goodhart (2009) and Giesecke and Kim (2009) propose measuring systemic risk via the probability that a "large" number of firms are in distress. Lucas, et al. (2011) use the same measure applied to European sovereign debt. Huang, et al. (2009) suggest using the price of a hypothetical contract insuring against system-wide distress, valued using a mix of CDS and equity data, as a measure of systemic risk. Schwaab (2010) presents a review of these and other measures of systemic risk.

We consider two different estimates of systemic risk, defined in detail in the following two subsections. In all cases we use the dynamic copula model that performed best in the previous section, 
namely the heterogeneous dependence factor copula model.

\subsection{Joint probability of distress}

The first measure of systemic risk we implement is an estimate of the probability that a large number of firms will be in distress, similar to the measure considered by Segoviano and Goodhart (2009), Giesecke and Kim (2009) and Lucas, et al. (2011). We define distress as a firm's one-year-ahead CDS spread lying above some threshold:

$$
D_{i, t+250} \equiv \mathbf{1}\left\{S_{i, t+250}>c^{*}\right\}
$$

We choose the threshold as the cross-sectional average of the $99 \%$ quantiles of the individual CDS spreads:

$$
c^{*}=\frac{1}{N} \sum_{i=1}^{N} c_{i}^{*}
$$

where $\operatorname{Pr}\left[S_{i t} \leq c_{i}^{*}\right]=0.99$

In our sample, the $99 \%$ threshold corresponds to a CDS spread of 339 basis points. Using equation (11) above, this threshold yields an implied probability of default (assuming LGD is 0.6) of 5.7\%. (The average CDS spread across all firms is 97 basis points, yielding an implied PD of 1.6\%.) We also considered a threshold quantile of 0.95, corresponding to a CDS spread of 245 basis points, and the results are qualitatively similar.

We use the probability of a large proportion of firms being in distress as a measure of systemic risk. Define the "joint probability of distress" as:

$$
J P D_{t, k} \equiv \operatorname{Pr}_{t}\left[\left(\frac{1}{N} \sum_{i=1}^{N} D_{i, t+250}\right) \geq \frac{k}{N}\right]
$$

where $k$ is a user-chosen threshold for what constitutes a "large" proportion of the $N$ firms. We use $k=30$, and the results corresponding to $k=20$ and $k=40$ are qualitatively similar.

With a fixed threshold for distress, such as that in equation (18), the average individual probability of distress will vary through time. It may thus be of interest, given our focus on systemic 
risk, to consider a scaled version of the JPD, to remove the influence of time variation in individual probabilities of distress. To this end, define:

$$
\begin{aligned}
S J P D_{t, k} & \equiv \frac{J P D_{t, k}}{\operatorname{AvgIPD_{t}}} \\
\text { where } A v g I P D_{t} & \equiv \frac{1}{N} \sum_{i=1}^{N} E_{t}\left[D_{i, t+250}\right]
\end{aligned}
$$

The JPD and SJPD estimates must be obtained via simulations from our model, and we obtain these using 10,000 simulations. Given the computational burden, we compute estimates only every 20 trading days (approximately once per month).

The estimated joint probability of distress and scaled joint probability of distress are presented in Figure 4. We see from the left panel that the JPD rose dramatically during the financial crisis of late 2008 -mid 2009, with the probability of at least 30 firms being in distress reaching around $80 \%$. This panel also reveals that a large part of this increase in JPD is attributable to an increase in the average individual probability of distress, which rose to nearly $50 \%$ in the peak of the financial crisis.

In the right panel we report the ratio of these two lines and obtain the scaled probability of distress. This can be thought of as a "multiplier" of individual distress, as it shows the ratio of joint distress to average individual distress. This ratio reached nearly two in the financial crisis. Interestingly, while this ratio fell in late 2009, it rose again in 2010 and in late 2011, indicating that the level of systemic risk implied by observed CDS spreads is substantially higher now than in the pre-crisis period.

\section{[ INSERT FIGURE 4 ABOUT HERE ]}

\subsection{Expected proportion in distress}

Our second measure of systemic risk more fully exploits the ability of our dynamic copula model to capture heterogeneous dependence between individual CDS spread changes. For each firm $i$, we compute the expected proportion of stocks in distress conditional on firm $i$ being in distress:

$$
E P D_{i, t} \equiv E_{t}\left[\frac{1}{N} \sum_{j=1}^{N} D_{j, t+250} \mid D_{i, t+250}=1\right]
$$


The minimum value this can take is $1 / N$, as we include firm $i$ in the sum, and the maximum is one. We use the same indicator for distress as in the previous section (equation (17)). This measure of systemic risk is similar in spirit to the CoVaR measure proposed by Adrian and Brunnermeier (2009), in that it looks at distress "spillovers" from a single firm to the market as a whole.

In Figure 5 below we summarize the results from the EPD estimates, and present the average, and $10 \%$ and $90 \%$ quantiles of this measure across the 100 firms in our sample. We observe that the average EPD is around $30 \%$ in the pre-crisis period, rising to almost $60 \%$ in late 2008 , and returning to around $40 \%$ in the last year of our sample. Thus this figure, like the JPD and SJPD plot in Figure 4, is also suggestive of a large increase in systemic risk around the financial crisis, and higher level of systemic risk in the current period than in the pre-crisis period.

\section{[ INSERT FIGURE 5 ABOUT HERE ]}

The expected proportion in distress measure enables us to identify firms that are more strongly correlated with market-wide distress than others. When the EPD is low for a given firm, it reveals that distress for that firm is not a signal of widespread distress, i.e., firm $i$ is more idiosyncratic. Conversely, when the EPD is high, it reveals that distress for this firm is a sign of widespread distress, and so this firm is a "bellwether" for systemic risk. To illustrate the information from individual firm EPD estimates, Table 6 below presents the top five and bottom five firms according to their EPD on three dates in our sample period, the first day (January 2, 2006), a middle day (January 26, 2009) and the last day (April 17, 2012). We note that SLM Corporation ("Sallie Mae", in the student loan business) appears in the "least systemic" group on all three dates, indicating that periods in which it is in distress are, according to our model, generally unrelated to periods

of wider distress. Marsh and McLennan (which owns a collection of risk, insurance and consulting firms) and Baxter International (a bioscience and medical firm) each appear in the "most systemic" group for two out of three dates.

Table 6 also provides information on the spread of EPD estimates across firms. At the start of our sample the least systemic firms had EPDs of 2 to 3, indicating that only one to two other firms are expected to be in distress when they are in distress. At the end of our sample the least 
systemic firms had EPDs of 8 to 12, indicating a wider correlation of distress even among the least correlated. A similar finding is true for the most systemic firms: the EPDs for the most systemic firms rise from $48-53$ at the start of the sample to $84-94$ at the end. Thus there is a general increase in the correlation between firm distress over this sample period.

\section{[ INSERT TABLE 6 ABOUT HERE]}

\section{Conclusion}

Motivated by the growing interest in measures of the risk of systemic events, this paper proposes new flexible yet parsimonious models for time-varying high dimension distributions. We use copula theory to combine well-known models for conditional means, variances, and marginal distributions with new models of the conditional dependence structure (copula) to obtain dynamic joint distributions. Our proposed new dynamic copula models draw on successful ideas from the literature on dynamic modeling of high dimension covariance matrices, see Engle (2002) and Engle and Kelly (2012) for example, and on recent work on models for general time-varying distributions, see Creal, et al. (2011, 2013), along with the "factor copula" of Oh and Patton (2012).

We use the proposed models to undertake a detailed study of a collection of 100 credit default swap (CDS) spreads on U.S. firms, which provide an relatively novel view of the health of these firms. We find, unsurprisingly, that systemic risk was highest during the financial crisis of 2008-09. More interestingly, we also find that systemic risk has remained relatively high, and is substantially higher now than in the pre-crisis period. 


\section{Appendix A: Obtaining the factor copula likelihood}

The factor copula introduced in Oh and Patton (2012) does not have a likelihood in closed form, but it is relatively simple to obtain the likelihood using numerical integration. Consider the factor structure in equation (5) and (6). Our objective is to obtain the copula density of $\mathbf{X}_{t}$.

$$
c_{t}\left(u_{1}, \ldots, u_{N}\right)=\frac{f_{x t}\left(G_{1 t}^{-1}\left(u_{1}\right), \ldots, G_{N t}^{-1}\left(u_{N}\right)\right)}{g_{1 t}\left(G_{1 t}^{-1}\left(u_{1}\right)\right) \cdots \cdot g_{N t}\left(G_{N t}^{-1}\left(u_{N}\right)\right)}
$$

where $f_{x t}\left(x_{1}, \ldots, x_{N}\right)$ is the joint density of $\mathbf{X}_{t}, g_{i t}\left(x_{i}\right)$ is the marginal density of $X_{i}$, and $c_{t}\left(u_{1}, \ldots, u_{N}\right)$ is the copula density. To construct copula density, we need each of the functions that appear on the right-hand side above: $g_{i t}\left(x_{i}\right), G_{i t}\left(x_{i}\right), f_{x t}\left(x_{1}, \ldots, x_{N}\right)$ and $G_{i t}^{-1}\left(u_{i}\right)$.

The independence of $Z$ and $\varepsilon_{i}$ implies that:

$$
\begin{aligned}
f_{X_{i} \mid Z, t}\left(x_{i} \mid z\right) & =f_{\varepsilon_{i}}\left(x_{i}-\lambda_{i t} z\right) \\
F_{X_{i} \mid Z, t}\left(x_{i} \mid z\right) & =F_{\varepsilon_{i}}\left(x_{i}-\lambda_{i t} z\right) \\
f_{\mathbf{X} \mid Z, t}\left(x_{1, \ldots,} x_{N} \mid z\right) & =\prod_{i=1}^{N} f_{\varepsilon_{i}}\left(x_{i}-\lambda_{i t} z\right)
\end{aligned}
$$

With these conditional distributions, one dimensional integration gives the marginals:

$$
g_{i t}\left(x_{i}\right)=\int_{-\infty}^{\infty} f_{X_{i}, Z, t}\left(x_{i}, z\right) d z=\int_{-\infty}^{\infty} f_{X_{i} \mid Z, t}\left(x_{i} \mid z\right) f_{Z, t}(z) d z=\int_{-\infty}^{\infty} f_{\varepsilon_{i}}\left(x_{i}-\lambda_{i t} z\right) f_{Z, t}(z) d z
$$

and similarly

$$
\begin{aligned}
G_{i t}\left(x_{i}\right) & =\int_{-\infty}^{\infty} F_{\varepsilon_{i}}\left(x_{i}-\lambda_{i t} z\right) f_{Z, t}(z) d z \\
f_{x t}\left(x_{1}, \ldots, x_{N}\right) & =\int_{-\infty}^{\infty} \prod_{i=1}^{N} f_{\varepsilon_{i}}\left(x_{i}-\lambda_{i t} z\right) f_{Z, t}(z) d z
\end{aligned}
$$

We use a change of variables, $U \equiv F_{Z, t}(z)$, to convert these to bounded integrals:

$$
\begin{aligned}
g_{i t}\left(x_{i}\right) & =\int_{0}^{1} f_{\varepsilon_{i}}\left(x_{i}-\lambda_{i t} F_{Z, t}^{-1}(u)\right) d u \\
G_{i t}\left(x_{i}\right) & =\int_{0}^{1} F_{\varepsilon_{i}}\left(x_{i}-\lambda_{i t} F_{Z, t}^{-1}(u)\right) d u \\
f_{x t}\left(x_{1}, \ldots, x_{N}\right) & =\int_{0}^{1} \prod_{i=1}^{N} f_{\varepsilon_{i}}\left(x_{i}-\lambda_{i t} F_{Z, t}^{-1}(u)\right) d u
\end{aligned}
$$


Thus the factor copula density requires the computation of just one-dimensional integrals. (For a factor copula with $J$ common factors the integral would be $J$-dimensional.) We use Gauss-Legendre quadrature for the integration, using $Q$ "nodes," (see Judd (1998) for details) and we choose $Q$ on the basis of a small simulation study described below.

Finally, we need a method to invert $G_{i t}\left(x_{i}\right)$, and note from above that this is a function of both $x$ and the factor loading $\lambda_{i t}$, with $G_{i t}=G_{j s}$ if $\lambda_{i t}=\lambda_{j s}$. We estimate the inverse of $G_{i t}$ by creating a grid of 100 points for $x$ in the interval $\left[x_{\min }, x_{\max }\right]$ and 50 points for $\lambda$ in the interval $\left[\lambda_{\min }, \lambda_{\max }\right]$, and then evaluating $G$ at each of those points. We then use two-dimensional linear interpolation to obtain $G^{-1}(u ; \lambda)$ given $u$ and $\lambda$. This two-dimensional approximation substantially reduces the computational burden, especially when $\lambda$ is time-varying, as we can evaluate the function $G$ prior to estimation, rather than re-estimating it for each likelihood evaluation.

We conducted a small Monte Carlo simulation to evaluate the accuracy of this numerical approximation. We use quadrature nodes $Q \in\{10,50,150\}$ and $\left[x_{\text {start }}, x_{\text {end }}\right]=[-30,30],\left[\lambda_{\text {start }}, \lambda_{\text {end }}\right]=$ $[0,6]$ for the numerical inversion. For this simulation, we considered the factor copula implied by the following structure:

$$
\begin{aligned}
X_{i} & =\lambda_{0} Z_{t}+\varepsilon_{i}, i=1,2 \\
\text { where } Z_{t} & \sim \operatorname{Skewt}\left(\nu_{0}, \psi_{0}\right), \varepsilon_{i t} \sim \operatorname{iid} t\left(\nu_{0}\right), Z \Perp \varepsilon_{i} \forall i
\end{aligned}
$$

where $\lambda_{0}=1, \nu_{0}^{-1}=0.25$ and $\psi_{0}=-0.5$. At each replication, we simulate $\mathbf{X}=\left[X_{1}, X_{2}\right] 1000$ times, and apply empirical distribution functions to transform $\mathbf{X}$ to $\mathbf{U}=\left[U_{1}, U_{2}\right]$. With this $\left[U_{1}, U_{2}\right]$ we estimate $\left[\lambda, \nu^{-1}, \psi\right]$ by numerically approximated maximum likelihood method.

Table S3 in the internet appendix contains estimation results for 100 replications. We find that estimation with only 10 nodes introduces a relatively large bias, in particular for $\nu^{-1}$, consistent with this low number of nodes providing a poor approximation of the tails of this density. Estimation with 50 nodes gives accurate results, and is comparable to those with 150 nodes in that bias and standard deviation are small. We use 50 nodes throughout the paper. 


\section{Appendix B: Proof of Proposition 1}

Proof of Proposition 1. (i) Consider the evolution equation for $\lambda_{i t}$ :

$$
\log \lambda_{i t}=\omega_{i}+\beta \log \lambda_{i, t-1}+\alpha s_{i, t-1}, \quad i=1,2, \ldots, N
$$

where $s_{i, t-1} \equiv \partial \log \mathbf{c}\left(\mathbf{u}_{t-1} ; \boldsymbol{\lambda}_{t-1}, \nu_{z}, \psi_{z}, \nu_{\varepsilon}\right) / \partial \lambda_{i, t-1}$. Creal, et al. (2013) show $E_{t-1}\left[s_{i, t}\right]=0$, so:

$$
E\left[\log \lambda_{i t}\right]=\omega_{i}+\beta E\left[\log \lambda_{i, t-1}\right]=\frac{\omega_{i}}{1-\beta}
$$

under stationarity of $\left\{\boldsymbol{\lambda}_{t}\right\}$, which holds by assumption 1 (b). So we have $\omega_{i}=E\left[\log \lambda_{i t}\right](1-\beta)$, and we can re-write our GAS equation in "variance targeting" form:

$$
\log \lambda_{i t}=E\left[\log \lambda_{i t}\right](1-\beta)+\beta \log \lambda_{i, t-1}+\alpha s_{i, t-1}
$$

The objective of this proposition is to find an estimate of $E\left[\log \lambda_{i t}\right]$ based on observable data.

Note that the linear correlation between $\left(X_{i}, X_{j}\right)$ is

$$
\begin{aligned}
\rho_{i j, X}^{L} & \equiv \operatorname{Corr}\left[X_{i}, X_{j}\right]=\frac{\lambda_{i} \lambda_{j}}{\sqrt{\left(1+\lambda_{i}^{2}\right)\left(1+\lambda_{j}^{2}\right)}} \equiv g\left(\lambda_{i}, \lambda_{j}\right) \\
\text { and } \mathbf{R}_{X}^{L} & \equiv \operatorname{Corr}[\mathbf{X}]=G(\boldsymbol{\lambda})
\end{aligned}
$$

By assumption 1(a), this is an exactly- $(N=3)$ or over- $(N>3)$ identified system, as we have $N$ parameters $\boldsymbol{\lambda} \equiv\left[\lambda_{1}, \ldots, \lambda_{N}\right]^{\prime}$ and $N(N-1) / 2$ correlations. Note that by Assumption $1(\mathrm{~d})$ we have a corresponding exactly- or over-identified system for the rank correlation matrix:

$$
\mathbf{R}_{X}=\varphi\left(\mathbf{R}_{X}^{L}\right)=\varphi(G(\boldsymbol{\lambda}))
$$

(In a slight abuse of notation, we let $\varphi\left(\mathbf{R}_{X}^{L}\right)$ map the entire linear correlation matrix to the rank correlation matrix.) Define the exponential of the inverse of the function $\varphi \circ G$ as $H$, so that $\log \boldsymbol{\lambda}=H\left(\boldsymbol{\rho}_{X}\right)$, where $\boldsymbol{\rho}_{X} \equiv \operatorname{vech}\left(\mathbf{R}_{X}\right)$. The function $H$ is not known in closed form but it can be obtained by a simple and fast optimization problem:

$$
H\left(\boldsymbol{\rho}_{X}\right)=\arg \min _{\mathbf{a}}\left(\operatorname{vech}\{\varphi(G(\exp \mathbf{a}))\}-\boldsymbol{\rho}_{X}\right)^{\prime}\left(\operatorname{vech}\{\varphi(G(\exp \mathbf{a}))\}-\boldsymbol{\rho}_{X}\right)
$$

This is the GMM analog to the usual method-of-moments estimator used in variance targeting. 
Under Assumption 1(c) the function $H\left(\boldsymbol{\rho}_{X}\right)$ is linear, so

$$
E\left[\log \boldsymbol{\lambda}_{t}\right]=E\left[H\left(\boldsymbol{\rho}_{t, X}\right)\right]=H\left(\overline{\boldsymbol{\rho}}_{X}\right)
$$

where $\overline{\boldsymbol{\rho}}_{X} \equiv E\left[\boldsymbol{\rho}_{t, X}\right]$.

Finally, we exploit the fact that RankCorr $[\mathbf{X}]$ is identical to RankCorr $[\boldsymbol{\eta}]$ by Assumption 1(a) and Theorem 5.1.6 of Nelsen (2006). So we obtain:

$$
E\left[\log \boldsymbol{\lambda}_{t}\right]=H\left(\overline{\boldsymbol{\rho}}_{X}\right)=H\left(\overline{\boldsymbol{\rho}}_{\eta}\right)
$$

(ii) We use as our "VT estimator" the sample analog of the above expression:

$$
\widehat{\widehat{\log \boldsymbol{\lambda}}}=H\left(\hat{\boldsymbol{\rho}}_{\eta}\right)
$$

First note that, since the marginal distributions of $\boldsymbol{\eta}_{t}$ are known, sample rank correlations are a linear functions of a sample moment, see Nelsen (2006, Chapter 5) for example:

$$
\hat{\rho}_{i j, \eta}^{S}=-3+\frac{12}{T} \sum_{t=1}^{T} F_{i}\left(\eta_{i, t}\right) F_{j}\left(\eta_{j, t}\right)
$$

Our estimate of $E\left[\log \lambda_{i t}\right]$ is obtained in equation (27) as:

$$
\begin{aligned}
\widehat{\widehat{\log \boldsymbol{\lambda}}} & =\arg \min _{\mathbf{a}} \overline{\mathbf{m}}_{T}(\mathbf{a})^{\prime} \overline{\mathbf{m}}_{T}(\mathbf{a}) \\
\text { where } \quad \overline{\mathbf{m}}_{T}(\mathbf{a}) & \equiv \operatorname{vech}\{\varphi(G(\exp \mathbf{a}))\}-\hat{\boldsymbol{\rho}}_{\eta}^{S}
\end{aligned}
$$

The element of $\overline{\mathbf{m}}_{T}$ corresponding to the $(i, j)$ element of the correlation matrix is:

$$
\bar{m}_{T}^{(i, j)}(\mathbf{a})=[\varphi(G(\exp \mathbf{a}))]_{(i, j)}+3-\frac{12}{T} \sum_{t=1}^{T} F_{i}\left(\eta_{i, t}\right) F_{j}\left(\eta_{j, t}\right)
$$

Thus $\widehat{\log \lambda}$ is a standard GMM estimator for $N \geq 3$. 


\section{References}

[1] Adrian, T. and M.K. Brunnermeier, 2009, CoVaR, Staff Report 348, Federal Reserve Bank of New York.

[2] Almeida, C., C. Czado, and H. Manner, 2012, Modeling high dimensional time-varying dependence using D-vine SCAR models, working paper, http://arxiv .org/pdf/1202.2008v1.pdf.

[3] Barclays Capital, 2010, Standard Corporate CDS Handbook.

[4] Blasques, F., S.J. Koopman and A. Lucas, 2012, Stationarity and Ergodicity of Univariate Generalized Autoregressive Score Processes, Tinbergen Institute Discussion Paper TI 2012$059 / 4$.

[5] Bollerslev, T., 1986, Generalized autoregressive conditional heteroskedasticity, Journal of Econometrics, 31, 307-327.

[6] Brownlees, C.T. and R.F. Engle, 2011, Volatility, Correlation and Tails for Systemic Risk Measurement, working paper, Stern School of Business, New York University.

[7] Carr, P. and L. Wu, 2011, A Simple Robust Link Between American Puts and Credit Protection, Review of Financial Studies, 24(2), 473-505.

[8] Chen, X. and Y. Fan, 2006, Estimation and model selection of semiparametric copula-based multivariate dynamic models under copula misspecification, Journal of Econometrics, 135, 125-154.

[9] Christoffersen, P., V. Errunza, K. Jacobs and H. Langlois, 2011, Is the potential for international diversification disappearing?, Review of Financial Studies, forthcoming.

[10] Christoffersen, P., K. Jacobs, X. Jin and H. Langlois, 2013, Dynamic Dependence in Corporate Credit, working paper, Bauer College of Business, University of Houston.

[11] Conrad, J., R.F. Dittmar and A. Hameed, 2011, Cross-Market and Cross-Firm Effects in Implied Default Probabilities and Recovery Values, working paper, Kenan-Flagler Business School, University of North Carolina.

[12] Creal, D.D., S.J. Koopman, and A. Lucas, 2011, A dynamic multivariate heavy-tailed model for time-varying volatilities and correlations, Journal of Business and Economic Statistics, 29 (4), 552-563.

[13] Creal, D.D., S.J. Koopman, and A. Lucas, 2013, Generalized Autoregressive Score Models with Applications, Journal of Applied Econometrics, forthcoming.

[14] Creal, D.D., R.B. Gramercy and R.S. Tsay, 2012, Market-based Credit Ratings, working paper, University of Chicago Booth School of Business.

[15] Davis, R. A., W. T. M. Dunsmuir, and S. Streett, 2003, Observation driven models for Poisson counts, Biometrika, 90(4), 777-790.

[16] Duffie, D. and K.J. Singleton, 2003, Credit Risk: Pricing, Measurement, and Management, Princeton University Press, New Jersey. 
[17] Engle, R.F., 1982, Autoregressive Conditional Heteroscedasticity with Estimates of the Variance of UK Inflation, Econometrica, 50, 987-1007.

[18] Engle, R.F., 2002, Dynamic conditional correlation: A simple class of multivariate generalized autoregressive conditional heteroskedasticity models, Journal of Business \& Economic Statistics, 20(3), 339-350.

[19] Engle, R.F. and B. Kelly, 2012, Dynamic Equicorrelation, Journal of Business and Economic Statistics, 30(2), 212-228.

[20] Engle, R.F. and J. Mezrich, 1996, GARCH for Groups. Risk, 9, 36-40.

[21] Engle, R.F. and J.R. Russell, 1998, Autoregressive Conditional Duration: A New Model for Irregularly Spaced Transaction Data, Econometrica, 66, 1127-1162.

[22] Engle, R.F. and K. Sheppard, 2001, Theoretical and Empirical Properties of Dynamic Conditional Correlation Multivariate GARCH, working paper, University of California, San Diego.

[23] Giesecke, K. and B. Kim, 2011, Systemic risk: What defaults are telling us, Management Science, 57(8), 1387-1405.

[24] Glosten, L.R., R. Jagannathan, D.E. Runkle, 1993, On the relation between the expected value and the volatility of the nominal excess return on stocks, Journal of Finance, 48(5), 1779-1801.

[25] Gonçalves, S. and H. White, 2002, The Bootstrap of the Mean for Dependent Heterogeneous Arrays, Econometric Theory, 18, 1367-1384.

[26] Gonçalves, S., U. Hounyo, A.J. Patton and K. Sheppard, 2013, Bootstrapping two-stage extremum estimators, working paper, Oxford-Man Institute of Quantitative Finance.

[27] Hafner, C.M. and H. Manner, 2011, Dynamic Stochastic Copula Models: Estimation, Inference and Applications, Journal of Applied Econometrics, forthcoming.

[28] Hansen, B.E., 1994, Autoregressive conditional density estimation, International Economic Review, 35(3), 705-730.

[29] Harvey, A.C., 2013, Dynamic Models for Volatility and Heavy Tails, Econometric Society Monograph 52, Cambridge University Press, Cambridge.

[30] Harvey, A.C. and G. Sucarrat, 2012, EGARCH models with fat tails, skewness and leverage, working paper CWPE 1236, Cambridge University.

[31] Huang, X., H. Zhou and H. Zhu, 2009, A framework for assessing the systemic risk of major financial institutions, Journal of Banking and Finance, 33(11), 2036-2049.

[32] Hull, J.C., 2012, Risk Management and Financial Institutions, Third Edition, John Wiley \& Sons, New Jersey.

[33] Jondeau, E. and M. Rockinger, 2006, The copula-GARCH model of conditional dependencies: An international stock market application, Journal of International Money and Finance, 25, $827-853$. 
[34] Judd, K.L, 1998, Numerical Methods in Economics, MIT Press, Cambridge, USA.

[35] Lucas, A., B. Schwaab and X. Zhang, 2011, Conditional Probabilities for Euro Area Sovereign Default Risk, Tinbergen Institute discussion paper, 11-176/2/DSF29.

[36] Manner, H. and J. Segers, 2011, Tails of correlation mixtures of elliptical copulas, Insurance: Mathematics and Economics, 48(1), 153-160.

[37] Markit Group, 2009, The CDS Big Bang: Understanding the Changes to the Global CDS Contract and North American Conventions, research report.

[38] Nelsen, R.B., 2006, An Introduction to Copulas, Second Edition, Springer, U.S.A.

[39] Oh, D.H. and A.J. Patton, 2012, Modelling Dependence in High Dimensions with Factor Copulas, working paper, Duke University.

[40] Oh, D.H. and A.J. Patton, 2013, Simulated method of moments estimation for copula-based multivariate models, Journal of the American Statistical Association, forthcoming.

[41] Patton, A.J., 2006, Modelling asymmetric exchange rate dependence, International Economic Review, 47(2), 527-556.

[42] Patton, A.J., 2012, Copula methods for forecasting multivariate time series, in G. Elliott and A. Timmermann (eds.), Handbook of Economic Forecasting, Volume 2, Elsevier, Oxford, forthcoming.

[43] Politis, D.N., and J.P. Romano, 1994, The Stationary Bootstrap, Journal of the American Statistical Association, 89, 1303-1313.

[44] Rémillard, B., 2010, Goodness-of-fit tests for copulas of multivariate time series, working paper.

[45] Schwaab, B., 2010, New Quantitative Measures of Systemic Risk, Financial Stability Review Special Feature E, European Central Bank.

[46] Segioviano, M.A. and C. Goodhart, 2009, Banking stability measures, IMF Working Paper $\mathrm{WP} / 09 / 4$.

[47] Shephard, N., 2005, Stochastic Volatility: Selected Readings, Oxford University Press, Oxford.

[48] Stöber, J. and C. Czado, 2012, Detecting regime switches in the dependence structure of high dimensional financial data, working paper, Technische Universität München.

[49] White, H., 1994, Estimation, Inference and Specification Analysis, Econometric Society Monographs No. 22, Cambridge University Press, Cambridge. 
Table 1: Simulation results

\begin{tabular}{|c|c|c|c|c|c|c|c|}
\hline & True & Bias & Std & Median & $90 \%$ & $10 \%$ & $\underset{(90 \%-10 \%)}{\operatorname{Diff}}$ \\
\hline & \multicolumn{7}{|c|}{ Panel A: Equidependence } \\
\hline$\omega$ & 0.000 & 0.005 & 0.015 & 0.001 & 0.027 & -0.003 & 0.030 \\
\hline$\alpha$ & 0.050 & 0.000 & 0.003 & 0.050 & 0.051 & 0.048 & 0.003 \\
\hline$\beta$ & 0.980 & 0.002 & 0.004 & 0.980 & 0.989 & 0.979 & 0.010 \\
\hline$\nu^{-1}$ & 0.200 & 0.001 & 0.006 & 0.200 & 0.206 & 0.195 & 0.010 \\
\hline \multirow[t]{2}{*}{$\psi_{z}$} & 0.100 & 0.005 & 0.017 & 0.100 & 0.118 & 0.097 & 0.021 \\
\hline & \multicolumn{7}{|c|}{ Panel B: Block equidependence } \\
\hline$\omega_{1}$ & -0.030 & 0.000 & 0.005 & -0.030 & -0.025 & -0.035 & 0.010 \\
\hline$\omega_{2}$ & -0.023 & -0.001 & 0.004 & -0.024 & -0.020 & -0.030 & 0.010 \\
\hline$\omega_{3}$ & -0.017 & 0.000 & 0.005 & -0.017 & -0.011 & -0.023 & 0.012 \\
\hline$\omega_{4}$ & -0.010 & 0.000 & 0.004 & -0.011 & -0.005 & -0.016 & 0.011 \\
\hline$\omega_{5}$ & -0.003 & 0.001 & 0.004 & -0.002 & 0.004 & -0.007 & 0.011 \\
\hline$\omega_{6}$ & 0.003 & 0.001 & 0.004 & 0.004 & 0.009 & 0.000 & 0.009 \\
\hline$\omega_{7}$ & 0.010 & 0.002 & 0.005 & 0.012 & 0.018 & 0.007 & 0.012 \\
\hline$\omega_{8}$ & 0.017 & 0.001 & 0.005 & 0.017 & 0.025 & 0.012 & 0.013 \\
\hline$\omega_{9}$ & 0.023 & 0.001 & 0.005 & 0.024 & 0.030 & 0.018 & 0.012 \\
\hline$\omega_{10}$ & 0.030 & 0.003 & 0.006 & 0.033 & 0.040 & 0.024 & 0.015 \\
\hline$\alpha$ & 0.050 & 0.001 & 0.005 & 0.051 & 0.057 & 0.045 & 0.012 \\
\hline$\beta$ & 0.980 & -0.001 & 0.002 & 0.978 & 0.981 & 0.976 & 0.004 \\
\hline$\nu^{-1}$ & 0.200 & -0.005 & 0.008 & 0.196 & 0.202 & 0.184 & 0.018 \\
\hline$\psi_{z}$ & 0.100 & 0.004 & 0.025 & 0.103 & 0.138 & 0.071 & 0.068 \\
\hline
\end{tabular}

Notes: This table presents results from the simulation study described in Section 3. Panel A contains results for the equidependence model and Panel B for the "block equidependence" model. 
Table 1: Simulation results (continued)

\begin{tabular}{crrrrrrr}
\hline & True & Bias & Std & Median & $90 \%$ & $10 \%$ & $\begin{array}{c}\text { Diff } \\
(90 \%-10 \%)\end{array}$ \\
\hline & \multicolumn{7}{c}{ Panel C: Heterogeneous dependence } \\
\cline { 2 - 8 } & & & & \\
$\omega_{1}$ & -0.030 & 0.004 & 0.017 & -0.022 & -0.005 & -0.052 & 0.047 \\
$\omega_{5}$ & -0.028 & 0.004 & 0.016 & -0.020 & -0.005 & -0.046 & 0.041 \\
$\omega_{10}$ & -0.025 & 0.002 & 0.016 & -0.019 & -0.005 & -0.041 & 0.036 \\
$\omega_{15}$ & -0.022 & 0.002 & 0.013 & -0.019 & -0.004 & -0.043 & 0.039 \\
$\omega_{20}$ & -0.019 & 0.002 & 0.011 & -0.015 & -0.003 & -0.033 & 0.030 \\
$\omega_{25}$ & -0.016 & 0.000 & 0.010 & -0.014 & -0.003 & -0.030 & 0.027 \\
$\omega_{30}$ & -0.012 & 0.001 & 0.008 & -0.010 & -0.002 & -0.022 & 0.020 \\
$\omega_{35}$ & -0.009 & 0.000 & 0.008 & -0.008 & -0.002 & -0.020 & 0.018 \\
$\omega_{40}$ & -0.006 & -0.001 & 0.005 & -0.006 & -0.002 & -0.015 & 0.014 \\
$\omega_{45}$ & -0.003 & -0.001 & 0.005 & -0.003 & 0.000 & -0.010 & 0.010 \\
$\omega_{50}$ & 0.000 & -0.002 & 0.004 & -0.002 & 0.001 & -0.007 & 0.008 \\
$\omega_{55}$ & 0.003 & -0.001 & 0.004 & 0.001 & 0.007 & -0.003 & 0.010 \\
$\omega_{60}$ & 0.006 & -0.002 & 0.005 & 0.003 & 0.010 & 0.000 & 0.010 \\
$\omega_{65}$ & 0.009 & -0.002 & 0.006 & 0.005 & 0.013 & 0.000 & 0.013 \\
$\omega_{70}$ & 0.012 & -0.004 & 0.007 & 0.007 & 0.017 & 0.001 & 0.016 \\
$\omega_{75}$ & 0.015 & -0.004 & 0.008 & 0.009 & 0.019 & 0.002 & 0.017 \\
$\omega_{80}$ & 0.018 & -0.004 & 0.009 & 0.012 & 0.026 & 0.002 & 0.024 \\
$\omega_{85}$ & 0.021 & -0.006 & 0.011 & 0.014 & 0.032 & 0.002 & 0.030 \\
$\omega_{90}$ & 0.024 & -0.006 & 0.012 & 0.016 & 0.036 & 0.003 & 0.033 \\
$\omega_{95}$ & 0.027 & -0.006 & 0.014 & 0.018 & 0.040 & 0.004 & 0.036 \\
$\omega_{100}$ & 0.030 & -0.007 & 0.016 & 0.021 & 0.040 & 0.004 & 0.036 \\
$\alpha$ & 0.050 & -0.006 & 0.015 & 0.045 & 0.062 & 0.023 & 0.039 \\
$\beta$ & 0.980 & 0.002 & 0.012 & 0.983 & 0.997 & 0.966 & 0.031 \\
$\nu_{-1}$ & 0.200 & -0.002 & 0.009 & 0.199 & 0.209 & 0.186 & 0.023 \\
$\psi_{z}$ & 0.100 & 0.008 & 0.032 & 0.111 & 0.152 & 0.064 & 0.088 \\
\hline & & & & & & & \\
\hline
\end{tabular}

Notes: This table presents results from the simulation study described in Section 3. Panel C contains results for the "heterogeneous dependence" model. In the interests of space, Panel C only reports every fifth intercept parameter $\left(\omega_{i}\right)$ rather than the complete set of 100 such parameters; the complete table is available in the internet appendix. 

Table 2: Summary statistics for daily CDS spreads
and log-differences of daily CDS spreads

\begin{tabular}{|c|c|c|c|c|c|c|}
\hline & Mean & $5 \%$ & $25 \%$ & Median & $75 \%$ & $95 \%$ \\
\hline \multicolumn{7}{|c|}{ Panel A: Cross-sectional distribution of CDS spreads } \\
\hline Mean & 96.953 & 37.212 & 53.561 & 74.957 & 123.785 & 200.346 \\
\hline Std dev & 69.950 & 17.344 & 27.245 & 47.508 & 84.336 & 180.618 \\
\hline $1^{\text {st }}$-order autocorrel & 0.996 & 0.992 & 0.995 & 0.997 & 0.998 & 0.998 \\
\hline Skewness & 1.203 & 0.095 & 0.695 & 1.280 & 1.587 & 2.488 \\
\hline Kurtosis & 5.113 & 2.198 & 2.943 & 4.937 & 6.477 & 9.486 \\
\hline $5 \%$ & 23.883 & 9.021 & 11.741 & 18.926 & 29.851 & 60.538 \\
\hline $25 \%$ & 42.274 & 20.373 & 25.212 & 35.314 & 47.473 & 104.704 \\
\hline Median & 85.310 & 35.098 & 50.105 & 69.399 & 113.762 & 166.208 \\
\hline $75 \%$ & 122.061 & 46.250 & 65.862 & 93.622 & 154.729 & 251.112 \\
\hline $95 \%$ & 245.497 & 72.514 & 102.554 & 168.500 & 313.585 & 631.924 \\
\hline $99 \%$ & 338.676 & 80.414 & 122.885 & 231.295 & 435.224 & 827.098 \\
\hline \multicolumn{7}{|c|}{ Panel B: Cross-sectional distribution of log-differences of CDS spreads } \\
\hline Mean & 5.589 & -1.634 & 2.559 & 5.529 & 8.521 & 13.817 \\
\hline Std dev & 378.892 & 308.636 & 347.627 & 373.460 & 400.385 & 476.533 \\
\hline $1^{\text {st }}$-order autocorrel & 0.161 & 0.030 & 0.121 & 0.164 & 0.217 & 0.267 \\
\hline Skewness & 1.087 & -0.285 & 0.354 & 0.758 & 1.488 & 3.629 \\
\hline Kurtosis & 25.531 & 7.717 & 10.286 & 14.557 & 25.911 & 74.843 \\
\hline $5 \%$ & -514.574 & -622.282 & -551.334 & -509.554 & -474.027 & -415.651 \\
\hline $25 \%$ & -144.195 & -172.319 & -155.635 & -145.415 & -134.820 & -111.993 \\
\hline Median & -2.324 & -9.045 & -3.644 & -0.726 & 0.000 & 0.000 \\
\hline $75 \%$ & 132.127 & 95.168 & 120.514 & 131.019 & 144.363 & 174.645 \\
\hline $95 \%$ & 570.510 & 457.775 & 537.093 & 568.331 & 612.769 & 684.984 \\
\hline \multicolumn{7}{|c|}{ Panel $C$ : Autocorrelation in $C D S$ spreads } \\
\hline \# of rejections & Level & Log-diff & Squared & $\log$-diff & & \\
\hline ADF test of unit root & 12 & 100 & - & & & \\
\hline LB test for autocorrel & - & 98 & 8 & & & \\
\hline
\end{tabular}

Notes: This table presents summary statistics of daily CDS spreads (upper panel) and logdifferences of CDS spreads (middle panel), measured in basis points in both cases. The columns present the mean and quantiles from the cross-sectional distribution of the measures listed in the rows. These two panels present summaries across the $\mathrm{N}=100$ marginal distributions. The bottom panel shows the number of rejections (at the 0.05 level) across the 100 firms for augmented DickeyFuller tests of the null of a unit root, as well as Ljung-Box tests for autocorrelation up to 10 lags. 
Table 3: Marginal distribution parameter estimates

\begin{tabular}{|c|c|c|c|c|c|c|}
\hline & \multicolumn{6}{|c|}{ Cross-sectional distribution } \\
\hline & Mean & $5 \%$ & $25 \%$ & Median & $75 \%$ & $95 \%$ \\
\hline$\phi_{0}$ & 3.029 & -3.760 & 0.247 & 3.116 & 5.861 & 10.165 \\
\hline$\phi_{1}$ & 0.005 & -0.179 & -0.062 & 0.010 & 0.082 & 0.153 \\
\hline$\phi_{2}$ & 0.025 & -0.039 & -0.001 & 0.025 & 0.050 & 0.084 \\
\hline$\phi_{3}$ & -0.002 & -0.058 & -0.028 & -0.004 & 0.021 & 0.064 \\
\hline$\phi_{4}$ & 0.006 & -0.046 & -0.014 & 0.006 & 0.033 & 0.054 \\
\hline$\phi_{5}$ & 0.004 & -0.055 & -0.022 & 0.005 & 0.027 & 0.060 \\
\hline$\phi_{m}$ & 0.387 & 0.163 & 0.303 & 0.372 & 0.480 & 0.638 \\
\hline$\omega / 1000$ & 5.631 & 1.401 & 3.111 & 5.041 & 7.260 & 13.381 \\
\hline$\beta$ & 0.741 & 0.595 & 0.699 & 0.746 & 0.794 & 0.845 \\
\hline$\alpha$ & 0.114 & 0.052 & 0.087 & 0.106 & 0.141 & 0.181 \\
\hline$\delta$ & 0.022 & 0.000 & 0.000 & 0.000 & 0.042 & 0.086 \\
\hline$\alpha_{m}$ & 0.223 & 0.037 & 0.137 & 0.206 & 0.297 & 0.494 \\
\hline$\delta_{m}$ & 0.072 & 0.000 & 0.000 & 0.059 & 0.114 & 0.233 \\
\hline$\nu$ & 3.620 & 2.877 & 3.293 & 3.571 & 3.921 & 4.496 \\
\hline \multirow[t]{2}{*}{$\psi$} & 0.043 & -0.003 & 0.024 & 0.042 & 0.062 & 0.089 \\
\hline & & & & & \multicolumn{2}{|c|}{ \# of rejections } \\
\hline \multicolumn{5}{|c|}{ LB test for standardized residuals } & \multicolumn{2}{|c|}{9} \\
\hline \multicolumn{5}{|c|}{ LB test for squared standardized residuals } & \multicolumn{2}{|c|}{2} \\
\hline \multicolumn{5}{|c|}{ KS test for skew $\mathrm{t}$ dist of std. residuals } & \multicolumn{2}{|c|}{11} \\
\hline
\end{tabular}

Notes: The table presents summaries of the estimated AR(5)-GJR-GARCH(1,1)-Skew $t(\nu, \psi)$ models estimated on log-difference of daily CDS spreads. The columns present the mean and quantiles from the cross-sectional distribution of the parameters listed in the rows. The bottom panel shows the number of rejections (at the 0.05 level) across 100 firms from Ljung-Box tests for serial correlation up to 10 lags. The first row is for standardized residuals of log-difference of daily CDS spreads and the second row for squared standardized residuals. The bottom panel shows the number of rejections across 100 firms from the Kolmogorov-Smirnov test of the Skew $t(\nu, \psi)$ distribution used for the standardized residuals. 

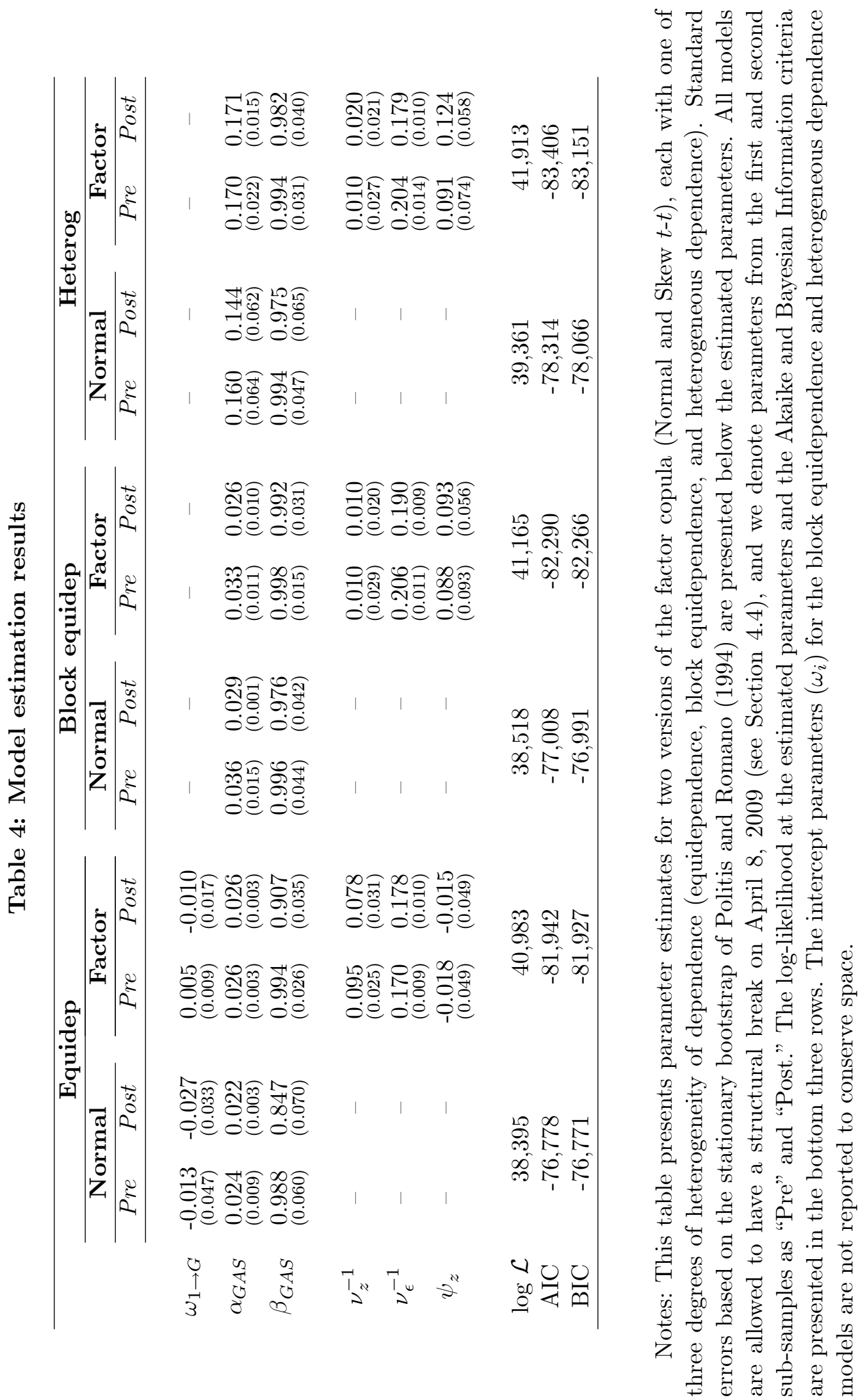
Table 5: Model comparison results

\begin{tabular}{|c|c|c|c|c|c|c|c|}
\hline & \multicolumn{2}{|c|}{ Normal } & \multicolumn{2}{|c|}{ Factor $\left(\nu, \psi_{z}, \nu\right)$} & \multicolumn{2}{|c|}{ Factor $\left(\nu_{z}, \psi_{z}, \nu_{\varepsilon}\right)$} \\
\hline & & Static & GAS & Static & GAS & Static & GAS \\
\hline \multirow{4}{*}{$\begin{array}{l}\text { Equi- } \\
\text { dependence }\end{array}$} & \# param & 2 & 6 & 6 & 10 & 8 & 12 \\
\hline & $\log \mathcal{L}$ & 36,185 & 38,395 & 39,223 & 40,688 & 39,508 & 40,983 \\
\hline & $\mathrm{AIC}$ & $-72,366$ & $-76,778$ & $-78,434$ & $-81,356$ & $-79,000$ & $-81,942$ \\
\hline & $\mathrm{BIC}$ & $-72,364$ & $-76,771$ & $-78,427$ & $-81,344$ & $-78,990$ & $-81,927$ \\
\hline \multirow{4}{*}{$\begin{array}{l}\text { Block } \\
\text { equi- } \\
\text { dependence }\end{array}$} & \# param & 10 & 14 & 14 & 18 & 16 & 20 \\
\hline & $\log \mathcal{L}$ & 36,477 & 38,518 & 39,441 & 40,799 & 39,757 & 41,165 \\
\hline & $\mathrm{AIC}$ & $-72,934$ & $-77,008$ & $-78,854$ & $-81,562$ & $-79,482$ & $-82,290$ \\
\hline & $\mathrm{BIC}$ & $-72,922$ & $-76,991$ & $-78,837$ & $-81,540$ & $-79,463$ & $-82,266$ \\
\hline \multirow{4}{*}{$\begin{array}{l}\text { Heterogeneous } \\
\text { dependence }\end{array}$} & \# param & 200 & 204 & 204 & 208 & 206 & 210 \\
\hline & $\log \mathcal{L}$ & 37,652 & 39,361 & 40,357 & 41,522 & 40,628 & 41,913 \\
\hline & $\mathrm{AIC}$ & $-74,904$ & $-78,314$ & $-80,306$ & $-82,628$ & $-80,844$ & $-83,406$ \\
\hline & $\mathrm{BIC}$ & $-74,661$ & $-78,066$ & $-80,058$ & $-82,375$ & $-80,594$ & $-83,151$ \\
\hline
\end{tabular}

Notes: This table presents the log-likelihood at the estimated parameters, as well as the Akaike and Bayesian Information criteria, for a variety of copula models. The preferred model according to each of these criteria is highlighted in bold. Also presented is the number of estimated parameters; note that this accounts for the fact that we allow for a structural break in these parameters, and so the number reported is twice as large as it would be in the absence of a break. We consider models with three degrees of heterogeneity of dependence (equidependence, block equidependence, and heterogeneous dependence); with and without dynamics (static and GAS); and three versions of the factor copula (Normal, Skew $t$ - $t$ with a common degrees of freedom parameter, and Skew $t$ - $t$ with separately estimated degrees of freedom parameters). 


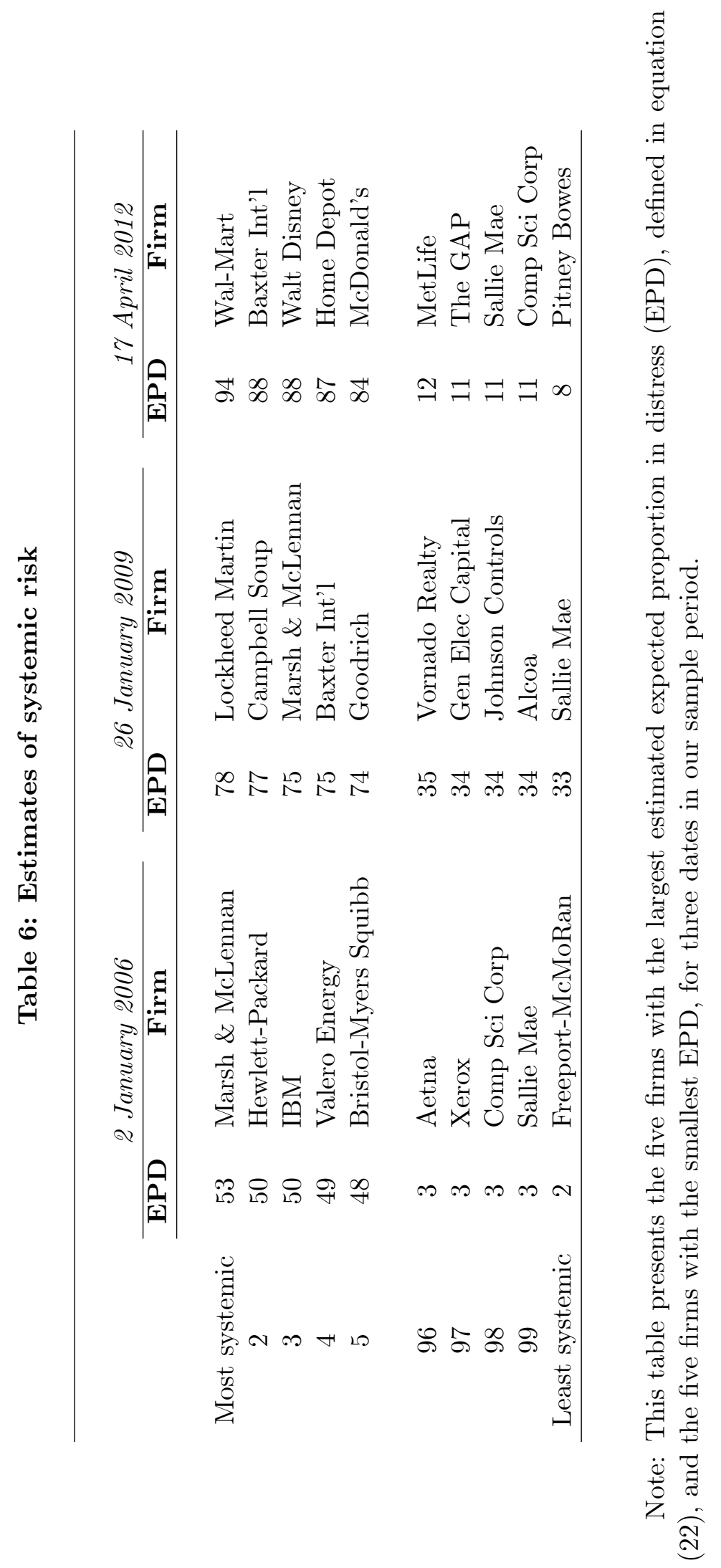



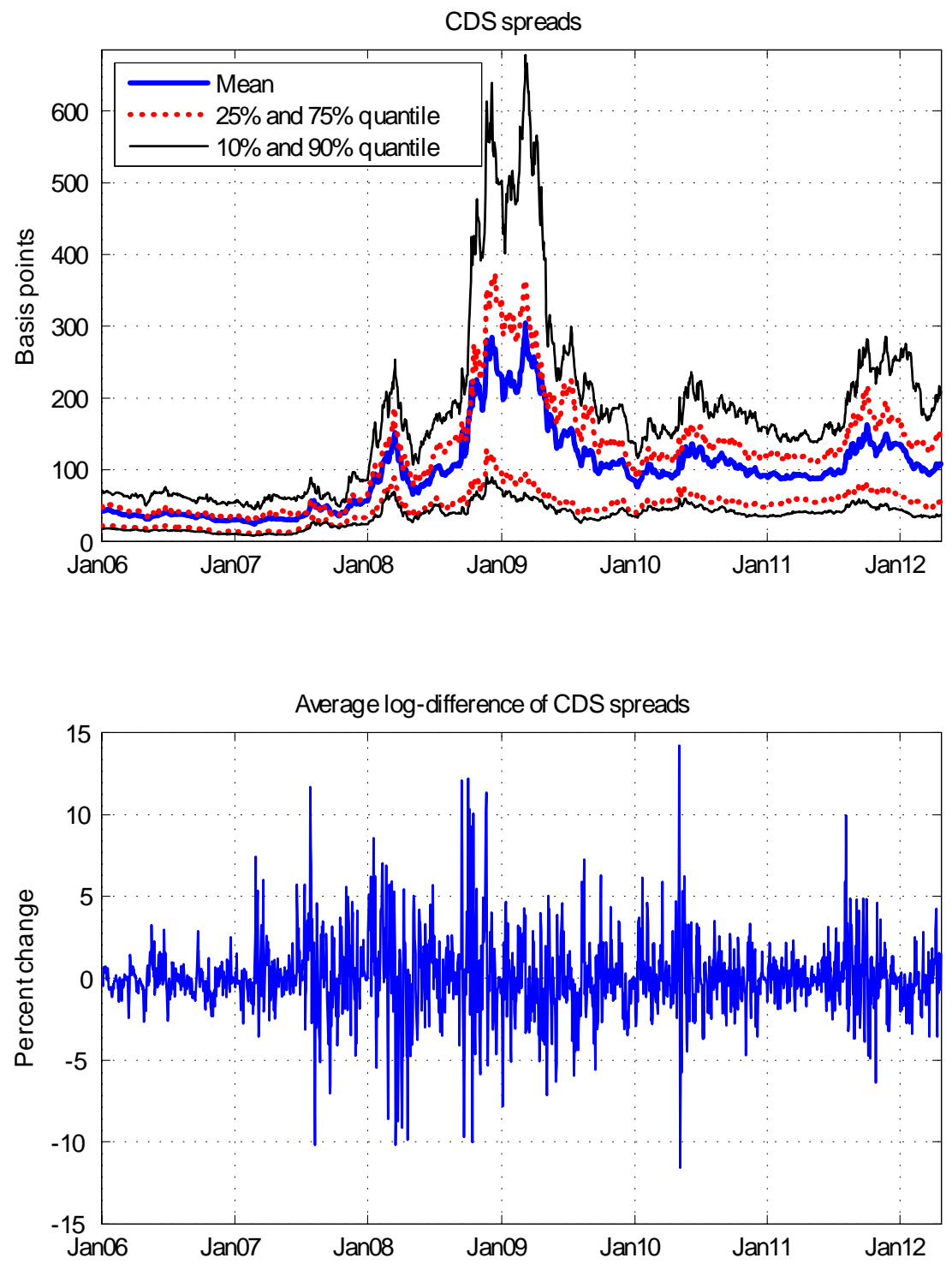

Figure 1: The upper panel plots the mean and 10\%, 25\%, $75 \%$ and $90 \%$ quantiles across the CDS spreads for 100 U.S. firms over the period January 2006 to April 2012. The lower panel reports the average (across firms) percent change in CDS spreads for the same time period. 


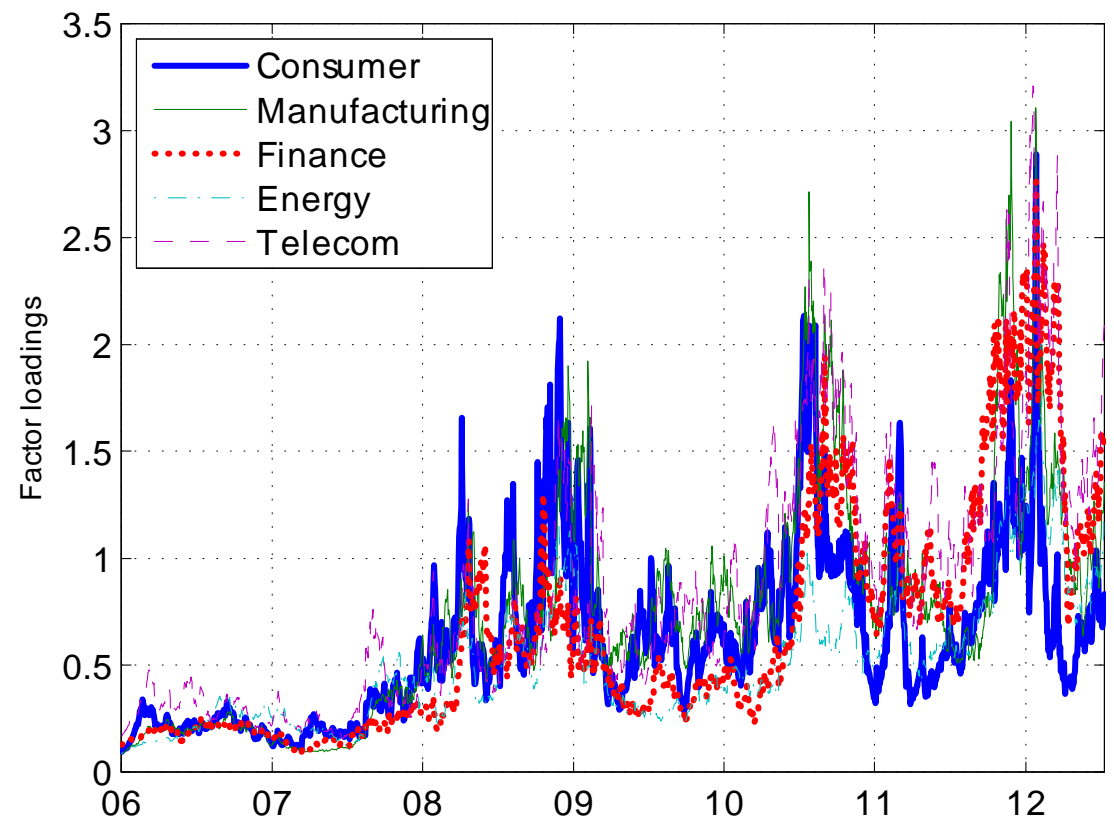

Figure 2: This figure plots the estimated factor loadings $\left(\lambda_{t}\right)$ from the heterogeneous dependence factor copula model, averaged across firms in the same industry. 

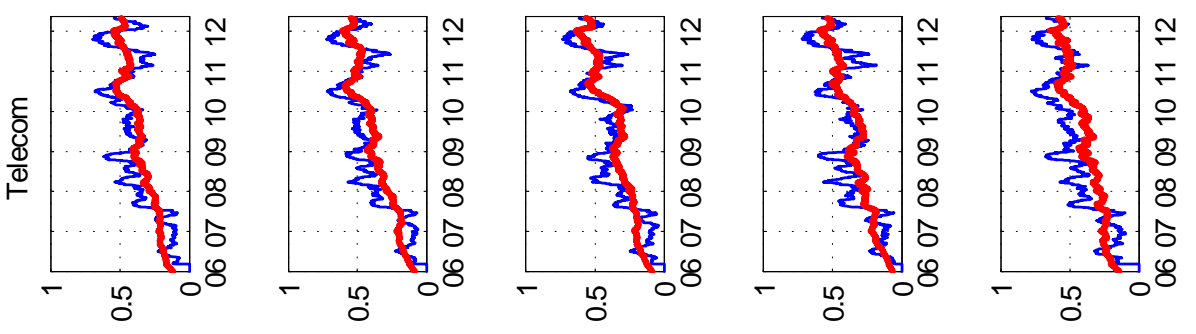

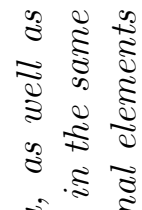

₹

के हैं :

ธิ ठิ

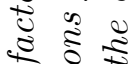

竎
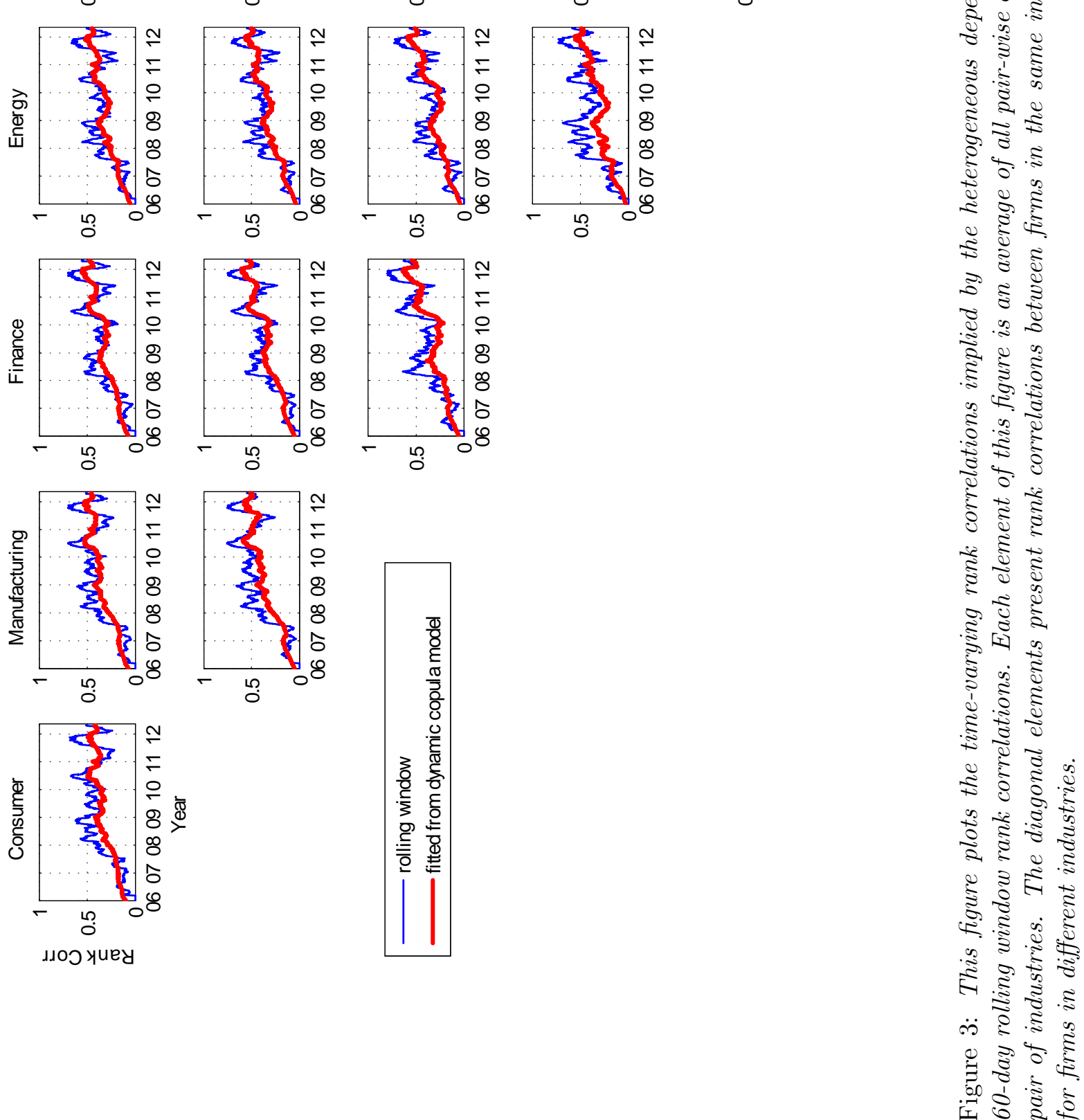

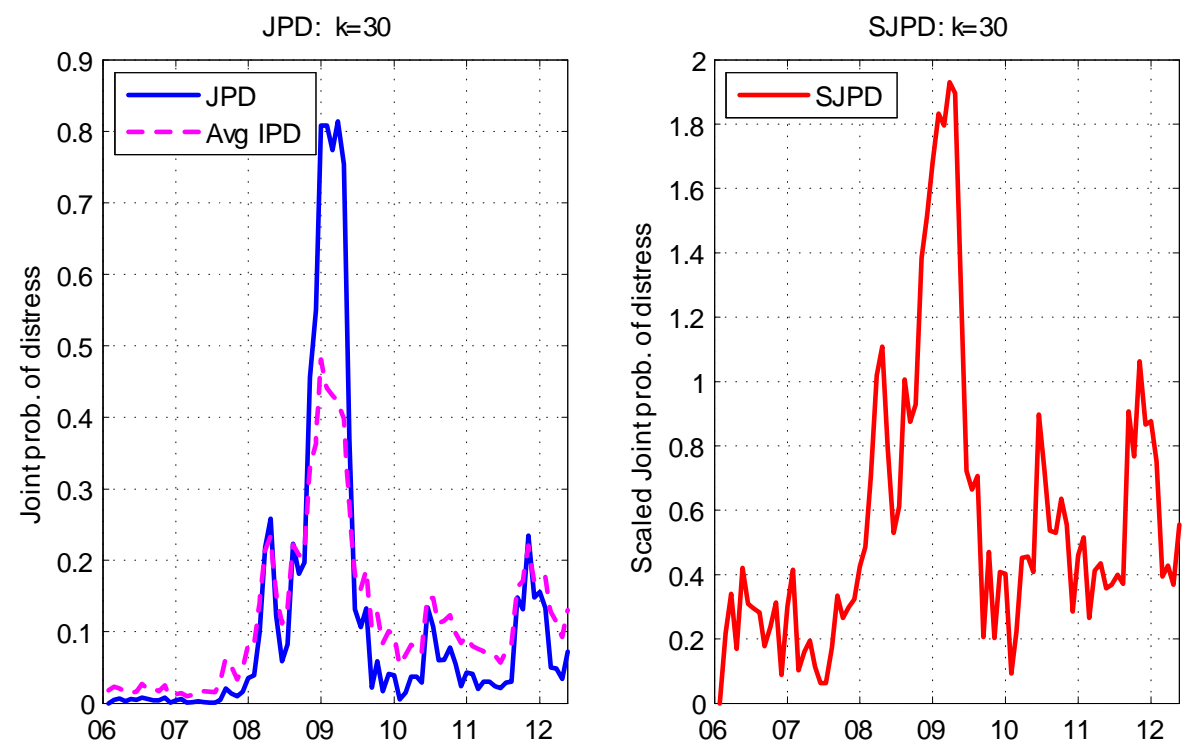

Figure 4: The left panel shows the joint probability of distress (JPD) in a solid line and the average individual probability of distress (Avg IPD) in a dashed line. The right panel shows the scaled joint probability of distress (SJPD). Both panels cover the period January 2006 to April 2012.

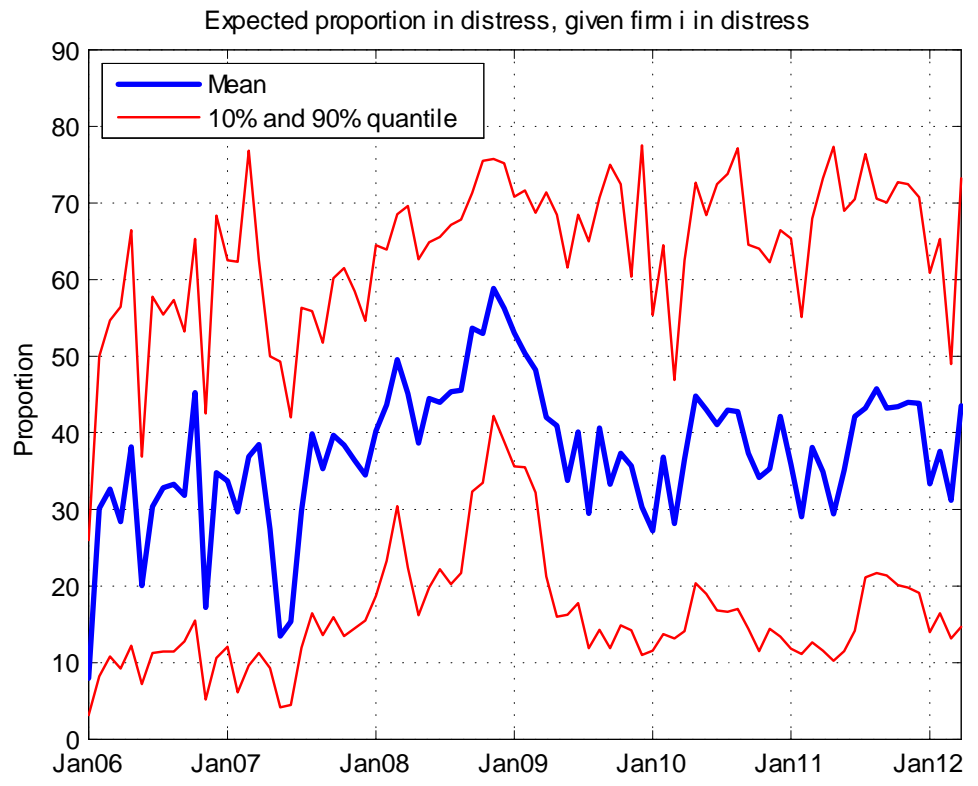

Figure 5: This figure shows the expected proportion (in percent) of firms in distress, given firm $i$ in distress, averaged across all 100 firms. The cross-sectional 10\% and 90\% quantiles are also reported. The sample period is January 2006 to April 2012. 